\title{
Generalizing Empirical Adequacy I: Multiplicity and Approximation
}

\author{
Sebastian Lutz* \\ Preprint: $2014-03-05$
}

\begin{abstract}
I provide an explicit formulation of empirical adequacy, the central concept of constructive empiricism, and point out a number of problems. Based on one of the inspirations for empirical adequacy, I generalize the notion of a theory to avoid implausible presumptions about the relation of theoretical concepts and observations, and generalize empirical adequacy with the help of approximation sets to allow for lack of knowledge, approximations, and successive gain of knowledge and precision. As a test case, I provide an application of these generalizations to a simple interference phenomenon.
\end{abstract}

Keywords: constructive empiricism; empirical adequacy; approximation; approximation set; empirical substructure; empirical embedding; empirical relativized reduct

\section{Contents}

\section{Introduction}

2 Measuring an interference phenomenon 2

2.1 Epistemic and approximate appearances . . . . . . . . 3

2.2 The improvement of epistemic and approximate appearances . . . 7

3 Empirical adequacy: Definitions, virtues, and problems 8

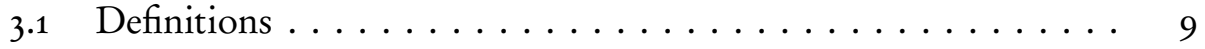

3.2 Virtues and problems ................ 12

*Munich Center for Mathematical Philosophy, Ludwig-Maximilians-Universität München. sebastian.lutz@gmx.net. A very early version of this paper has been presented at Herman Philipse's Dutch Research Seminar in Analytic Philosophy at Utrecht University. Many thanks to the participants and Anthony Booth, Otávio Bueno, and Thomas Müller for very helpful discussions, and two anonymous referees for helpful comments. Many of the definitions are inspired by, but differ significantly from those given by Bueno (1997). Part of the research for this article was supported by the Alexander von Humboldt Foundation. 
4 Approximation sets 16

5 Generalizations 19

5.1 Empirical relativized reducts $\ldots \ldots \ldots \ldots \ldots \ldots \ldots$

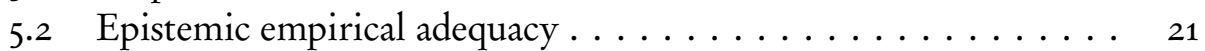

5.3 Approximate empirical adequacy $\ldots \ldots \ldots \ldots \ldots \ldots \ldots$

5.4 Hierarchies of empirical adequacy $\ldots \ldots \ldots \ldots \ldots \ldots \ldots$

6 Conclusion 29

$\begin{array}{lr}\text { A Proofs } & 29\end{array}$

In Memory of Marian Przetęcki

\section{Introduction}

At the core of constructive empiricism lies the concept of empirical adequacy. For according to van Fraassen (1980, 12, emphasis removed), constructive empiricism is the view that

[s]cience aims to give us theories which are empirically adequate; and acceptance of a theory involves as belief only that it is empirically adequate.

The model theoretic notion of empirical adequacy that van Fraassen uses is very strict: It does not allow for approximation, nor does it allow for lack of knowledge of the phenomena. What is more, the definition puts enormous restrictions on the structures of scientific theories $(\$ 3.2)$.

In this article, I suggest a generalization of van Fraassen's notion of a theory that alleviates van Fraassen's unduly restrictive demands (\$5.1), and I suggest generalizations of his notion of empirical adequacy that allow for lack of knowledge of the phenomena $(\$ 5.2)$, for approximation $(\$ 5.3)$, and for an increase of our knowledge about the phenomena $(\$ 5.4)$. The basis for these generalizations is provided by one of the inspirations for van Fraassen's definition of empirical adequacy, the work by Polish model theoretician Marian Przełęcki (\$4). To show the viability of the generalizations, I apply them to the investigation of a simple interference phenomenon, which I will present first.

\section{Measuring an interference phenomenon}

In an informal overview, van Fraassen $(1980,12)$ states that "a theory is empirically adequate exactly if what it says about the observable things and events in this world, is true", where what is observable is described by the theory " $T$ itself, and the theories used as auxiliaries in the testing and application of $T$ " (57). 
Call the observable events 'phenomena' and their descriptions 'appearances'. Van Fraassen $(1980,19)$ stresses that the notion of empirical adequacy "will have to be spelt out very carefully if it is not to bite the dust among hackneyed objections" and I will spell out van Fraassen's notion in detail in the next section, but as a rough guide through my example, the informal notion will do. For one, it already shows that empirical adequacy is a categorical concept, since what a theory says about the phenomena is either true or not, and so the theory is either empirically adequate or it is not. As van Fraassen $(1991,12)$ puts it: "Empirical adequacy, like truth, admits of no degrees". This leads to problems when the empirical adequacy of a scientific theory is investigated. As will become clear in the example, empirical adequacy can describe one important aspect of scientific research, but it is too crude a concept to describe a number of similarly important aspects.

\subsection{Epistemic and approximate appearances}

Take an application of the ray theory of light. In the ray theory, light beams are taken to be rays that propagate according to Fermat's principle of stationary time, that is, they take a path whose infinitesimal variation leaves the propagation time invariant. Additionally, each ray is assigned an intensity as an additive magnitude. Now assume that two light beams of the same intensity travel in a homogeneous medium on the same line in opposite directions. According to Fermat's principle, the line will be straight, and so one can describe the intensity of the light beams as a function of one spatial Cartesian coordinate $x$, even though the beams have non-vanishing thickness. The ray theory further asserts that the intensities of the two beams will simply add up to some intensity $\psi^{\text {ray }}(x)=I$ that is constant for all $x$ and at all times, so that the time averaged intensity is also constant for all $x$ :

$$
\overline{\psi^{\text {ray }}}(x)=I \text {. }
$$

$\overline{\psi^{\text {ray }}}$ then describes the phenomenon of two light beams superimposed for some time (say, at least a few seconds) according to the ray theory of light. For the purposes of this example, I will assume that the phenomenon is in fact correctly described by the wave theory of light. Then the beams actually produce an interference pattern

$$
\bar{\psi}(x)=2 I \cos ^{2}\left(\frac{2 \pi x}{\lambda}\right)=I+I \cos \left(\frac{4 \pi x}{\lambda}\right)
$$

for the time averaged intensity (cf. Batterman 2002, \$6.2).

To measure the brightness of the light beams, one could tilt them ever so slightly, not enough to lead to significant changes in the calculation above, but enough so that they illuminate a screen placed parallel and in close enough proximity to their path. This is possible because the beams have non-vanishing thickness. A scientist could visually compare the brightness of the interference pattern at different spatial locations on the screen and thus come up with relative 


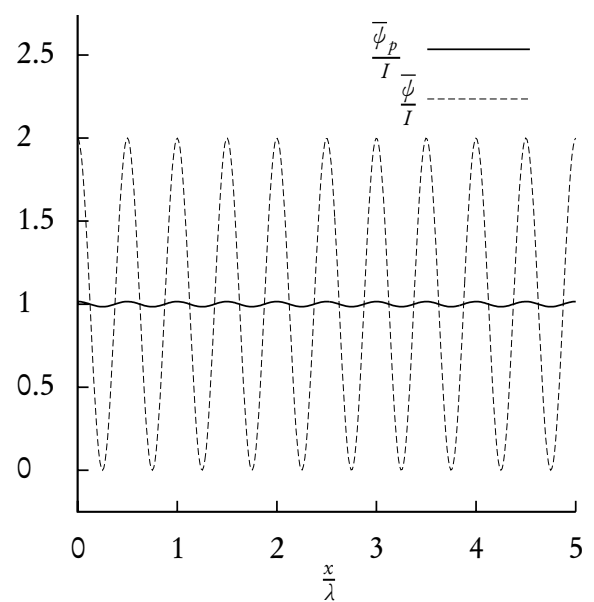

Figure 1: The pattern of the intensity according to the wave theory (dashed) and the blurred pattern (solid) when a finite spatial resolution $p / \lambda=10.25$ of the eye is taken into account.

intensity values for the two beams for different spatial locations $x$. This requires no special measuring devices, which would need to be described by additional theories. It does, however, require a theory about the scientist's eyes, which provide access to the phenomenon. For the purposes of this example, I will assume a very simple auxiliary theory according to which the scientist's eyes perceive brightness as linearly correlated with intensity and with a finite resolution, and furthermore have some finite spatial resolution. ${ }^{1}$

For measurement devices in general, finite resolutions can often be described by some normalized function $b$, so that the measurement of $\bar{\psi}$ will be spatially blurred to the convolution $\bar{\psi}_{b}(x)=\int_{-\infty}^{\infty} \bar{\psi}(y) b(y-x)$ dy. Assuming, for ease of calculation, that $b$ is a rectangular function of width $p$ centered around 0 , the convolution amounts to an averaging over the spatial interval $[x-p / 2, x+p / 2]$. The measurement of $\bar{\psi}$ then gives the result

$$
\bar{\psi}_{p}(x)=\frac{1}{p} \int_{x-\frac{p}{2}}^{x+\frac{p}{2}} 2 I \cos ^{2}\left(\frac{2 \pi y}{\lambda}\right) \mathrm{d} y=I+\frac{I \lambda}{2 \pi p} \sin \left(\frac{2 \pi p}{\lambda}\right) \cos \left(\frac{4 \pi x}{\lambda}\right)
$$

(figure 1). Assuming a finite resolution $q$ of the intensity as well, the measurement results in a function

$$
\bar{\psi}_{p}^{q}: \mathbb{R} \longrightarrow \wp \mathbb{R}^{\geq 0}, x \mapsto\left[\bar{\psi}_{p}(x)-\frac{q}{2}, \bar{\psi}_{p}(x)+\frac{q}{2}\right] \cap \mathbb{R} \geq 0
$$

that takes values for spatial locations to ranges of non-negative values for intensity.

1. Note that this auxiliary theory could also be assumed to describe a measurement device. This assumption would arguably fit better with scientific practice and would not require any modification of the formalism or the subsequent calculations. However, it would go against the distinguished status that constructive empiricism confers upon human perception. 
Now assume the scientist's eyes have under optimal conditions a spatial resolution $p_{\min }$ and an intensity resolution $q_{\min }$, so that the interference pattern as it appears to the scientist is described by $\bar{\psi}_{p_{\min }{ }^{2}}{ }^{2}$ The interference phenomenon is an observable event, and the scientist's description of the relative brightness is an appearance. The scientist's measurement report, however, will have to state a finite set of values, as Suppes (1962) famously pointed out. The measurement of the intensity pattern $\bar{\psi}_{p_{\min }}$ can thus be taken to result in some finite set

$$
\left\{\left\langle x_{1}, \bar{\psi}_{p_{\min }}^{q_{\min }}\left(x_{1}\right)\right\rangle, \ldots,\left\langle x_{s}, \bar{\psi}_{p_{\min }}^{q_{\min }}\left(x_{s}\right)\right\rangle\right\}
$$

of pairs of spatial values $x_{i}$ and intensity ranges $\bar{\psi}_{p_{\min }}^{q_{\min }}\left(x_{i}\right)$.

Now, the ray theory states that the brightness appears constant in different spatial locations, $\overline{\psi^{\text {ray }}}=I$, so that for any resolution $p$ the blurred intensity is given by

$$
{\overline{\psi^{\text {ray }}}}_{p}(x)=\frac{1}{p} \int_{x_{i}-\frac{p}{2}}^{x_{i}+\frac{p}{2}} I=I,
$$

which, with the finite intensity resolution $q$, leads to

$$
{\overline{\psi_{p}^{\text {ray }}}}_{p}^{q}: \mathbb{R} \longrightarrow \wp \mathbb{R}^{\geq 0}, x \mapsto\left[I-\frac{q}{2}, I+\frac{q}{2}\right] \cap \mathbb{R}^{\geq 0}
$$

Assuming that the ray theory can be combined with the same simple auxiliary theory about the scientist's eyes as the wave theory, the interference phenomenon according to the ray theory is then described by by $\frac{\psi_{p_{\min }}^{\text {ray }} q_{\min }}{p}=[I-$ $q / 2, I+q / 2]$. Hence $\overline{\psi_{p_{\min }}^{\text {ray }} q_{\min }} \neq \bar{\psi}_{p_{\min }}^{q_{\min }}$, and many of the data sets (5) reported by the scientists will contain pairs $\left\langle x_{i}, Y_{i}\right\rangle$ with $Y_{i} \neq \bar{\psi}_{p_{\min }}^{q_{\min }}\left(x_{i}\right)$. Hence what the ray theory says about the observable things or events in this world is false, and thus it is not empirically adequate. As far as empirical adequacy is concerned, this is the end of the ray theory, and there is nothing else to say. Constructive empiricism then demands that the ray theory must not be accepted.

But of course there is a lot more to say. For one, it might be that the scientist checking the empirical adequacy of the ray theory has not performed this specific experiment, and what ray theory asserts about those experiments that she has performed (say, measurements of the average intensities of single beams) is true. In this case, the ray theory is empirically adequate as far as she knows. Second, even if this specific experiment is performed, the scientist may not be in the right situation to realize that what the ray theory asserts about the intensity is false. In sub-optimal circumstances (e. g., when looking at the screen from a distance or at

2. Clearly, these are radically simplifying assumptions about the eye's physiology, but a more realistic account would complicate things considerably without leading to philosophical insights relevant for the following discussion. 


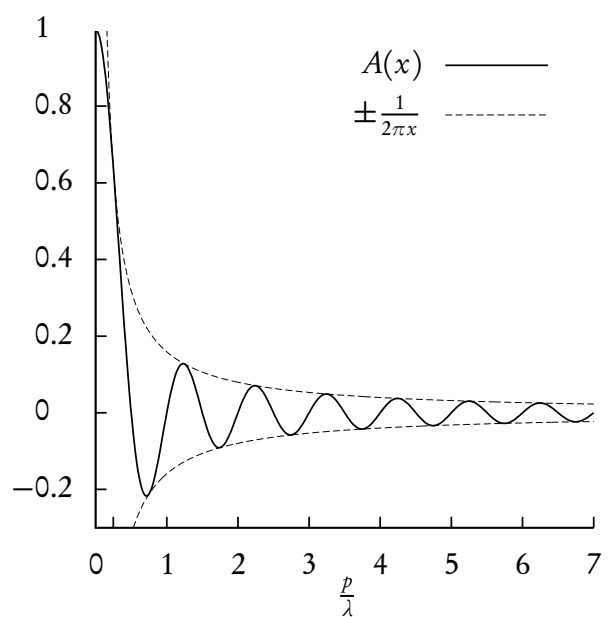

Figure 2: The deviation factor $D(x)$ (solid) enclosed between $\pm 1 /(2 \pi x)$ (dashed).

an angle, or if the laboratory is brightly lit), the spatial and intensity resolutions of the scientist's eyes will be lower, and hence $p>p_{\min }$ and $q>q_{\min }$. An increase of $q$ simply increases the interval of the intensity values (4). To see the effect of a variation of $p$, it is helpful to rewrite the blurred interference pattern (3) as

$$
\bar{\psi}_{p}(x)=I+I D\left(\frac{p}{\lambda}\right) \cos \left(\frac{4 \pi x}{\lambda}\right)
$$

with the deviation factor

$$
D(x)=\frac{\sin (2 \pi x)}{2 \pi x} .
$$

$D$ and the intensity $I$ determine how far individual values of the measured intensity $\bar{\psi}_{p}$ can maximally deviate from $I$, the intensity's spatial average. The maximal deviation thus depends solely on $I$ and the ratio of the precision of spatial measurement and the wavelength, $p / \lambda$. As can be seen from both the graph of $D$ (figure 2) and the formula (9) for $D$, the functions $1 /(2 \pi x)$ and $-1 /(2 \pi x)$ enclose $D .^{3}$

For intensities $I$ and wavelengths $\lambda$ small enough, as well as spatial resolutions and intensity resolutions low enough (i. e., $q$ and $p$ large enough), it will be difficult or impossible for the scientist to tell from $\bar{\psi}_{p}^{q}$ that the phenomenon is described by $\bar{\psi}_{p_{\min }}^{q_{\min }}$, not $\overline{\psi_{p_{\min }}^{\text {ray }}} q_{\min }$. This is because she only has access to epistemic appearances (described formally in definition 9), here given by $\bar{\psi}_{p}^{q}$, which only restrict which appearances are epistemically possible (definition 11), that is, possible as far as she knows. As long as her knowledge about the actual appearances (those under the best conditions) is incomplete, her epistemic appearances do not uniquely determine what the actual appearances are. In the example, both $\bar{\psi}_{p_{\text {min }}}^{q_{\min }}$

3. Note that $|D(x)| \leq 1$ also for $x \in[0,1 /[2 \pi])$, unlike $1 /(2 \pi x)$. 
and $\overline{\psi^{\text {ray }}} q_{\min }$ may be epistemically possible. In this case, for her the ray theory of light is epistemically empirically adequate (definition 10). Clearly, an epistemically empirically adequate theory does not always turn out to be empirically adequate as well; if and only if the the scientist can exclude all but one intensity pattern does she know the actual appearances (described formally by claim 4 ).

The example also provides a very practical sense in which the ray theory is approximately empirically adequate (definition 13): Although not empirically adequate simpliciter, the ray theory is empirically adequate for certain, but not all appearances and only up to certain resolutions, which leads to approximate appearances (definition 12). Approximately empirically adequate theories like the ray theory of light, quantum field theory, and general relativity are clearly useful, and for this reason, van Fraassen $(1989,366$, n. 5) himself suggests, but does not explicate, the notion of an "approximate embedding".

\subsection{The improvement of epistemic and approximate appearances}

The scientist who, on epistemic grounds, accepts the ray theory for now can of course still doubt its actual empirical adequacy. To assuage or confirm her doubts, she may over time improve her measurements of the interference phenomenon by, for instance, moving closer to the screen, looking at it at a right angle, training her ability to distinguish between differently bright spots, or simply by including more measurements in her report. This would lead to a restricted bierarchy of epistemic appearances (definition 15), where at each level, the epistemic appearances describe the phenomena better than the previous epistemic appearances. As Bueno $(1997,603)$ puts it, the scientist develops a hierarchy of appearances "built in such a way that, at each level, there is a gain of information regarding the phenomena being modeled". But such a hierarchy would be restricted to the phenomena currently under investigation, while in general, the scientist will also want to increase the number of phenomena she studies. This would lead to an (unrestricted) bierarchy of epistemic appearances (definition 14). As a special case, the scientist may develop restricted and unrestricted hierarchies of approximate appearances (definition 17) in which the appearances are closer and closer approximated (claim 6). All such hierarchies are best conceived of as possibly infinite, so that, for example, the epistemic or approximate appearances can asymptotically approach the appearances as they would be determined under optimal conditions. ${ }^{4}$

When moving up in the hierarchy, the information about the phenomena increases, and a theory that is not epistemically empirically adequate at some level of the hierarchy should not be epistemically empirically adequate at any higher level of the hierarchy either (claim 5). When focusing on a fixed set of phenomena (i.e., within a restricted hierarchy), one can also establish something of a converse: If at one point in a hierarchy, a theory is empirically adequate given all

4. I thank Leszek Wroński for this point. 
epistemically possible appearances, then it will be epistemically empirically adequate no matter how the hierarchy develops further (claim 7). New phenomena, of course, may still prove a theory empirically inadequate.

The increase of information about the phenomena in a hierarchy of epistemic appearances also allows for a comparative concept of epistemic empirical adequacy (definition 16). While van Fraassen $(1989,67)$ provides the comparative notion of one theory being "empirically at least as strong" as another, this concept serves a different purpose: If a theory $T_{1}$ is at least as strong as another theory $T_{2}$, then $T_{1}$ is empirically adequate only if $T_{2}$ is (claim 9), but that says nothing about the theories' actual empirical adequacy. Indeed, there is a negative correlation between between comparative empirical strength and comparative empirical adequacy: If a theory $T_{1}$ is empirically at least as strong as another theory $T_{2}$, then $T_{2}$ is at least as epistemically empirically adequate as $T_{1}$ no matter the hierarchy (claims 8 and 9). In other words, no matter how the scientist improves her information about the appearances, a theory that is empirically at least as strong as another will never be the second to be proven empirically inadequate.

The concepts introduced so far will be defined precisely in the following, which will also allow for proofs of the claims of this section. To this end, I will first spell out the definitions given by van Fraassen (1989) explicitly and discuss their features. For the new concepts, I will deviate as little as possible from van Fraassen's formalism; specifically, I will rely on one of the formalisms that inspired van Fraassen's definitions.

\section{Empirical adequacy: Definitions, virtues, and prob- lems}

Although van Fraassen (1980) defines empirical adequacy in terms of model theory, his formal exposition is rather light. I will thus rely on the standard notation as used by Chang and Keisler $(1990, \$ 1.3)$ and, more loosely, by Hodges (1993, $\mathbb{S} \mathbb{S} 1.2-1.3)$. Hence a structure $\mathfrak{A}$ is a pair $\langle A, \mathscr{I}\rangle$ consisting of a domain $A$ and a function $\mathscr{I}$ from a set of $m_{i}$-place relation symbols $R_{i}, n_{j}$-place function symbols $F_{j}$, and constant symbols $c_{k}$ to, respectively, $m_{i}$-ary relations, $n_{j}$-ary functions, and constants on $A$. Unless stated otherwise, I will in the following always assume this set of symbols with the same arities. I will sometimes refer to non-logical symbols as 'terms' when this does not lead to ambiguity. Sometimes, I use indexed structures $\mathfrak{M}_{i}$ instead of $\mathfrak{A}, \mathfrak{B}$, etc. $A$ will always be the domain $|\mathfrak{A}|$ of $\mathfrak{A}, B=|\mathfrak{B}|$ etc. If $\mathfrak{A}=\langle A, \mathscr{I}\rangle$, I write $R_{i}^{\mathfrak{A}}$ instead of $\mathscr{I}\left(R_{i}\right)$, and analogously for functions and constants. $R_{i}^{\mathfrak{B}}$ is the relation in $\mathfrak{B}$ that corresponds to relation $R_{i}^{\mathfrak{A}}$ in $\mathfrak{A}$, and analogous for functions and constants. In displayed form, I write a structure $\mathfrak{A}$ as $\left\langle A, R_{1}^{\mathfrak{A}}, \ldots, R_{s}^{\mathfrak{A}}, F_{1}^{\mathfrak{A}}, \ldots, F_{t}^{\mathfrak{A}}, c_{1}^{\mathfrak{A}}, \ldots, c_{u}^{\mathfrak{A}}\right\rangle$ or, for possibly infinite index sets, $\left\langle A, R_{i}^{\mathfrak{A}}, F_{j}^{\mathfrak{A}}, c_{k}^{\mathfrak{A}}\right\rangle_{i \in I, j \in J, k \in K}$. 
In contradistinction to the above, Bell and Slomson $(1974,73)$ define a relational structure $\mathfrak{A}$ as a pair $\left\langle A,\left\{R_{i}\right\}_{i<\alpha}\right\rangle$ of a domain and a set of relations, where $\alpha$ is a cardinal. This difference is little more than notational, since in their definitions of further model theoretic concepts, the corresponding relations are determined by the index set $\{i: i<\alpha\}$, which therefore plays the role of the set of relation symbols $\left\{R_{i}: i \in I\right\}$ used by Chang and Keisler $(1990,19-20)$ and Hodges $(1993,2)$. For examples relevant in the following, compare the definitions of reduct, isomorphism, and substructure by Chang and Keisler (1990, 20-23) and by Bell and Slomson $(1974,153,73)$, respectively. ${ }^{5}$ The reader who prefers the notation by Bell and Slomson (1974) will have no problems translating the following discussion.

\subsection{Definitions}

Within constructive empiricism, van Fraassen $(1980,64)$ states,

$[\mathrm{t}$ ]o present a theory is to specify a family of structures, its models; and secondly, to specify certain parts of those models (the empirical substructures) as candidates for the direct representation of observable phenomena.

Furthermore the models of the theory "are describable only up to structural isomorphism” (van Fraassen 2008, 238; cf. 2002, 22). More formally, this can be phrased as follows:

Definition 1. A theory $\left\langle\left\{\mathfrak{T}_{n}\right\}_{n \in N},\left\{\mathbf{E}_{n}\right\}_{n \in N}\right\rangle$ contains a family of structures (the models of the theory) and for each structure $\mathfrak{T}_{n}=\left\langle T_{n}, P_{i}^{\mathfrak{T}_{n}}, F_{j}^{\mathfrak{T}_{n}}\right.$, $\left.c_{k}^{\mathfrak{T}_{n}}\right\rangle_{i \in I_{n}, j \in J_{n}, k \in K_{n}}$ a set $\mathbf{E}_{n}$ of empirical substructures, such that for each $\mathfrak{E} \in \mathbf{E}_{n}$, $\mathfrak{E} \subseteq \mathfrak{T}_{n}$. With each model, a theory also contains every isomorphic structure and its corresponding ${ }^{6}$ empirical substructures.

As a shorthand, define $\left\langle\mathfrak{T}_{n}, \mathbf{E}_{n}\right\rangle:=\left\langle\left\{\mathfrak{T}_{n}\right\}_{n \in N},\left\{\mathbf{E}_{n}\right\}_{n \in N}\right\rangle$. Van Fraassen (1989, 16) distinguishes strictly between observable and unobservable entities, and furthermore between phenomena and appearances: "Phenomena are observable entities (objects, events, processes,...) of any sort, appearances are the contents of measurement outcomes" (van Fraassen 2008, 283). In the example with the light

5. For reasons that are not entirely clear, this notational convention has become a philosophical point of both contention and confusion. Van Fraassen $(1989$, 366, n. 4), for example, objects to structures being "yolked to a particular syntax", where 'syntax' seems to stand for 'set of nonlogical symbols in the object language' (see n. 15). And French and Ladyman $(1999,115)$ see support for van Fraassen's position in the definition of 'structure' given by Hodges (1993), which, however, assigns a particular set of non-logical symbols to each structure (cf. Lutz 2014b, $\$ 3$ ).

6. To be precise: If $f: T_{m} \longrightarrow T_{n}$ is an isomorphism between $\mathfrak{T}_{m}$ and $\mathfrak{T}_{n}$, then the set $\mathbf{E}_{n}$ of empirical substructures that corresponds to $\mathbf{E}_{m}$ contains all and only those structures $\mathfrak{E}$ for which there is an $\mathfrak{E}^{\prime} \in \mathbf{E}_{m}$ such that $f$ is an isomorphism between $\mathfrak{E}^{\prime}$ and $\mathfrak{E}$. 
beams, the screen and individual areas on the screen are observable (they are objects), and so are the events of their illumination. Indeed, the illumination of the screen as a whole is an observable event, and hence a phenomenon. That these objects and events are observable is a fact of human physiology, and thus observer relative; human perception has a distinguished status in constructive empiricism (van Fraassen 1980, 57). As is assumed in the use of a convolution function in the example, the objects' and events' observability can be described by a theory, and typically different theories describe what is observable and how it is observable in different ways. But since their observability is an empirical, objective fact, this does not make their observability theory relative (van Fraassen 1989, 57). By contrast, thus van Fraassen (2008, 284),

the appearance is determined jointly by the measurement set-up (involving both apparatus and the system to which it is applied), the experimental practice, and the theoretical conceptual framework in which the target and measurement procedure are classified, characterized, and understood.

This is clear in the example of the interference phenomenon: The phenomenon is described in terms of intensity and spatial position, which occurr in the conceptual frameworks of both the ray and the wave theory. The measurement set-up and the experimental practice (the direction of the light beams, the screen, and the scientist using her eyes) then determines the appearance in this framework. According to van Fraassen $(1980,64)$, appearances can be described by structures as well: "The structures which can be described in experimental and measurement reports we can call appearances". This suggests

Definition 2. Appearances are given by a set $\mathrm{A}$ of structures. A structure $\mathfrak{A} \in \mathbf{A}$ is an appearance.

Note that the set of appearances does not have to be closed under isomorphism. The appearances $\mathbf{A}$ in definition 2 must be taken as, first, relative to the group $g$ of persons whose perceptual apparatus is described by the theory $T$ that is used for determining what is observable, and second, relative to $T$. Formally, however, this would only result in two additional indices ( ' $\mathbf{A}_{g, T}$ ') that are constant as long as the same group and the same theory are discussed; hence I will suppress the indices in the following. But care has to be taken when dealing with two theories (or more) at once: When determining their empirical adequacy, one can use the same phenomena for both theories, but not generally the same appearances. In the example of the interference phenomenon, the use of the same appearance is only possible because, first, the same auxiliary theory was used, and second, the ray theory and the wave theory rely on the same terms that furthermore have the same extensions in the description of the interference phenomenon. The terms' intensions (and their conceptual role) in the respective theories may still differ, however. ${ }^{7}$

7. I thank an anonymous reviewer for this analysis. 
Van Fraassen $(1980,64)$ now defines a theory to be "empirically adequate if it has some model such that all appearances are isomorphic to empirical substructures of that model" (cf. van Fraassen 1991, 12):

Definition 3. A theory $\left\langle\mathfrak{T}_{n}, \mathbf{E}_{n}\right\rangle$ is empirically adequate for appearances $\mathbf{A}$ if and only if there is some $n \in N$ such that for every $\mathfrak{A} \in \mathbf{A}$, there is an $\mathfrak{E} \in \mathbf{E}_{n}$ with $\mathfrak{E} \cong \mathfrak{A}$.

Comparing van Fraassen's technical explication of empirical adequacy with his informal description shows that the former implicitly contains two further technical explications. For one, "what [a theory] says about the observable things" is determined by its empirical substructures, and second, the truth of those statements is the same as each appearance being isomorphic to one of those empirical substructures. As simplifying slogans, one could thus say that restricted aboutness is expressed by the substructure relation and truth is expressed by isomorphism.

Definition 3 defines the empirical adequacy of a theory relative to a set of appearances. In contradistinction, empirical adequacy simpliciter is defined as empirical adequacy for the set of all appearances (cf. Monton and Mohler 2008, $\$ 1.5)$. And it is for empirical adequacy simpliciter that van Fraassen (1980, 12, emphasis removed) claims that "acceptance of a theory involves as belief only that it is empirically adequate". Definitions 3 can thus already take a certain lack of knowledge about the appearances into account, since A may not contain all appearances. Such an incomplete set $\mathbf{A}$ of appearances may allow the deductive inference that some theory is not empirically adequate, but the inference that a theory is empirically adequate will have to be in some way ampliative.

A note on terminology: Van Fraassen $(1980,66)$ and others (e. g., Suárez 2005, \$4.1; Monton and Mohler 2008, $\mathbb{S} 1.5-1.6)$ occasionally speak of the empirical adequacy of a theory as the embeddability of the appearances into a model of the theory. But the two are not equivalent: $\mathfrak{A} \in \mathbf{A}$ can be embedded in $\mathfrak{T}_{n}$ if and only if $\mathfrak{A}$ is isomorphic to any substructure of $\mathfrak{T}_{n}$ (Hodges 1993, 6). The substructure does not have to be an empirical substructure. ${ }^{8}$ In the following, I will call an isomorphic mapping to an empirical substructure an empirical embedding.

Some exponents of empirical adequacy (e.g. Suárez 2005, 39) rely on a modified notion of empirical adequacy:

Definition 4. A theory $\left\langle\mathfrak{T}_{n}, \mathrm{E}_{n}\right\rangle$ is idiosyncratically empirically adequate for appearances $\mathbf{A}$ if and only if for every $\mathfrak{A} \in \mathbf{A}$, there are an $n \in N$ and an $\mathfrak{E} \in \mathbf{E}_{n}$ such that $\mathfrak{E} \cong \mathfrak{A}$.

8. In contradistinction, Muller (1997, 2) defines an empirical substructure to be any substructure that is isomorphic to an appearance. He therefore assumes that a theory has no distinguished empirical substructures independently of the appearances. (As Muller points out, if there is only one appearance that is relevant for the theory and the theory is empirically adequate according to Muller's definition, the theory has exactly one empirical substructure. If there are more appearances, that same theory can have more empirical substructures.) 
Definitions 3 and 4 are equivalent if there is only one appearance, $\mathbf{A}=\{\mathfrak{A}\},{ }^{9}$ but not in general (claim 1). ${ }^{10}$ It is easy to see that if $\left\langle\mathfrak{T}_{n}, \mathbf{E}_{n}\right\rangle$ is empirically adequate, then it is idiosyncratically empirically adequate, so that empirical adequacy is strictly stronger than idiosyncratic empirical adequacy. ${ }^{11}$

Since theories are closed under isomorphism, an appearance is empirically embeddable in a model of a theory if and only if it is an empirical substructure of a model of that theory (Hodges 1993, ex. 1.2.4b). Therefore a theory is idiosyncratically empirically adequate if and only if all appearances are empirical substructures of models of the theory (that is, in definition $4, \mathfrak{E} \cong \mathfrak{A}$ could be exchanged for $\mathfrak{E}=\mathfrak{A})$. This is not the case for empirical adequacy, however (claim 2).

\subsection{Virtues and problems}

Empirical adequacy has two important virtues: First, while constructive empiricism crucially relies on empirical adequacy, the reverse is not true. Even a realist can rely on empirical adequacy as one property of a theory, for example by inferring that some theory is false because it is not empirically adequate. In this sense, empirical adequacy is metaphysically neutral, but can be used to define an anti-realist position like constructive empiricism.

Second, while constructive empiricism confers a distinguished status upon human perception, the formalism describing empirical adequacy does not. It can therefore be used to describe empirical adequacy even if what counts as an observation is not determined by the scientist's eyes but, say, the measurement devices available to the scientist. ${ }^{12}$ Thus the formalism that van Fraassen has developed is to some extent independent of his epistemological positions.

These virtues, however, are countered by a significant shortcoming: A theory is empirical adequate if and only if everything it says about all appearances is completely true. It is not clear whether there is a single non-trivial scientific theory that fulfills this requirement, and not clear whether there ever will be. ${ }^{13}$

9. This is decidedly not what van Fraassen in general assumes (personal email from June 15, 2011), and it is also incompatible with his definitions of appearances and empirical adequacy quoted above: The "structures" (plural) given by measurements are appearances, and in the case of empirical adequacy, "all appearances are isomorphic to empirical substructures" (plural) of a single model. Some philosophers of science, e. g. Przełęcki, have claimed (independently of constructive empiricism) that the appearances are plausibly always expressible in a single structure; others, e. g. Sneed and Wójcicki, have defended the opposite (see Przełęcki 1974a, 103, and references therein).

10. If the family $\left\{\mathbf{E}_{n}\right\}_{n \in N}$ is understood as determined by the appearances (see n. 8) and 'substructure' is taken to mean 'relativized reduct' (see $\$ 5.1$ ), Muller $(1997,2)$ also relies on idiosyncratic empirical adequacy. Thus while his discussion of the relation of wave and matrix mechanics "can be regarded [...] as a morsel of applied philosophy of science", it cannot be regarded an application of van Fraassen's philosophy of science.

11. Since Suárez (2005) argues that van Fraassen's notion of empirical adequacy is too strong, his use of idiosyncratic empirical adequacy thus does not threaten his conclusion.

12. See n. 1.

13. The qualifier 'non-trivial' is essential: A theory that says next to nothing about anything 
The example of the interference phenomenon has made clear that an analysis of scientific research needs concepts that apply even if the theory's statements are not completely true. These concepts, I suggest, are epistemic and approximate empirical adequacy. The discussion of van Fraassen's formal notions has brought out the need for a concept that applies even if not all appearances have been taken into account. Such a concept is already given by definition 3, which defines empirical adequacy relative to some possibly incomplete set of appearances. Van Fraassen's notion is recovered by demanding empirical adequacy relative to all appearances. Without these concepts, empirical adequacy is only equipped to describe a small part of scientific research, namely the final state of measurement procedures, when all phenomena are described with maximum precision and without any uncertainties that could be removed by improved measurements. This state would be completely static, so that empirical adequacy provides no means of describing the development of the scientific testing of theories. For this, I suggest hierarchies of epistemic and approximate appearances.

Finally, there should be a concept that applies even if not everything a theory says about observable things is true. Specifically, not all concepts of a theory should have to be considered. This is especially clear for functions and constants. For it follows from the definition of a substructure that every constant of a model $\mathfrak{T}_{n}$ has to be in the domain $E$ of each of its substructures $\mathfrak{E} \in \mathbf{E}_{n}$. Furthermore, every function of the model $\mathfrak{E} \in \mathrm{E}_{n}$ must map all (tuples of) elements of $E$ to elements of $E$ (Hodges 1993, lemma 1.2.2). Thus if, for example, there is a successor function on the whole domain of the theory, every model $\mathfrak{T}_{n}$ has only itself as a (trivial) substructure. Therefore it can also have at most itself as empirical substructure. If now a theory $\left\langle\mathfrak{T}_{n}, \mathbf{E}_{n}\right\rangle$ is empirically adequate, every appearance is a substructure of some $\mathfrak{T}_{n}$, so that $\mathfrak{T}_{n}$ 's domain $T_{n}$ contains measurement results; hence all constants of $\mathfrak{T}_{n}$ are directly measurable, and all functions of $\mathfrak{T}_{n}$ map measurement results to other measurement results. It seems clear that few actual scientific theories meet these restrictions.

One may object to these problems for two reasons. For one, in early works van Fraassen $(1970, \$ 3)$ relied on "elementary statements" and a "satisfaction function" to give the relation between a theory and observations, so that one could argue that the model theoretic formalization above does not capture van Fraassen's position. However, van Fraassen $(1989,365$, n. 34) himself states that he soon "found it much more advantageous to concentrate on the propositions expressible by elementary statements, rather than on the statements themselves". Thus van Fraassen had abandoned the reliance on elementary statements and satisfaction functions by the time he defined empirical adequacy. More importantly, empirical adequacy is defined without reference to either of the two concepts, and thus an analysis of empirical adequacy does not have to take them into account

is likely to be empirically adequate (Worrall 1984, \$3; Percival 2007, 87; Lutz 2014a, \$6), but restricting scientific research to such theories would be cold comfort. Note that empirical adequacy is still a weaker requirement than truth, which would amount to the existence of an isomorphism between one of the models of the theory and (the structure of) the world. 
either.

One may also object that the terms 'embedding', 'substructure', and 'isomorphism' are not meant literally, but refer to relations between theories and phenomena given by either satisfaction functions or something completely different. Possible support comes from van Fraassen's standard example of embedding, the seven point geometry $(1980, \mathbb{3} .1 ; 1989, \$ 9.1)$, which is not an embedding in the sense of model theory (Turney 1990, 441-443). But this looks more like an oversight than a conscious decision. Furthermore, the terms are well-defined within, but not outside of model theory, where they also do not in general occur together. And the objection makes van Fraassen use these terms in a different, undefined way without pointing this out. This would be very problematic for van Fraassen's overall position of constructive empiricism, as he himself states that the notion of empirical adequacy must be spelled out carefully in order to avoid hackneyed criticisms. And so, without a carefully spelled out notion of its core concept, constructive empiricism may fall prey to the same criticisms.

The objection that van Fraassen does not use model theoretic terminology with its literal meaning also flies in the face of van Fraassen's own elaborations. For instance, van Fraassen (1980, 43-44) states explicitly that in his discussion of the embedding relationship between models, defined as one model "being isomorphic to a part (substructure)" of another, the use of the word 'model' "derives from logic and meta-mathematics".

The objection furthermore renders nonsensical many of van Fraassen's arguments. Take, for example, van Fraassen's position that syntactic accounts of empirical adequacy and equivalence have failed (\$3.6). In a counterargument, Worrall $(1984,71)$ points out that "to every normal ('elementary') set of models there corresponds a consistent set of first-order sentences" and argues that for this and other reasons, semantic approaches are not in principle superior to syntactic ones. Similarly, Friedman (1982, 276-277) argues that for elementary classes of models, "the Completeness Theorem immediately yields the equivalence of van Fraassen's account and the traditional syntactic account". Van Fraassen (1989, 211, n. 31) replies with a result from formal model theory (Bell and Slomson 1974, 141, lemma 1.13), stating that "when a theory is presented by defining the class of its models, that class of structures cannot generally be identified with an elementary class of models of any first-order language", that is, "the family of models may not be an elementary class". Thus van Fraassen counters Worrall and Friedman by pointing out that their arguments presume that all classes of models of theories are elementary (can be defined in predicate logic of first order), which, he states, is not generally the case. But if van Fraassen did not think of models in terms of formal model theory, he would have to respond that both critics are missing the point, since the completeness of first order logic (which connects syntactic descriptions only to structures of formal model theory) would be completely irrelevant for his claim that models in his sense cannot be described or analyzed syntactically. And the same would hold for the possibility of describing classes of structures of formal model theory, whether elementary or not, by first 
order sentences. In short, he would have to respond that Worrall and Friedman are trading on the ambiguity of 'model' when using results from model theory, but instead uses such results himself. ${ }^{14}$

Finally, the objection that van Fraassen does not use model theoretic terminology with its literal meaning would leave many of van Fraassen's claims unjustified. For example, van Fraassen $(1980,43)$ discusses cases in which "every model of $T_{1}$ can be embedded in (identified with a substructure of) a model of $T_{2}$." The parenthetical equivalence claim relies on the model theoretic definition of 'embedding' and 'substructure', on the closure of the set of models of a theory under isomorphism, and the equivalence of embedding and the substructure relation for classes of structures that are closed under isomorphism (Hodges 1993, ex. 1.2.4b). If the terms were not meant in the model theoretic sense, there would be no reason for this equivalence claim. Thus van Fraassen's conception of empirical adequacy relies crucially on model theoretic notions and the problems that result from a literal reading of his model theoretic definitions are indeed problems. ${ }^{15}$

There is, however, at least one alleged problem of van Fraassen's notion of empirical adequacy that poses no difficulty. It is based on Suppes's point that measurement results are reported as finite sets of data. Van Fraassen (2008, 166172) takes Suppes's point into account and makes a distinction between surface models, which for the interference phenomenon is given by $\bar{\psi}_{p_{\min }}^{q_{\min }}$, and data models, the measurement results. He then demands that "the data or surface models must ideally be isomorphically embeddable in theoretical models" (168). In their "cardinality objection", Bueno, French, and Ladyman $(2002,503)$ argue that such a demand is problematic because the domains of data structures "in general are finite". The implicit assumption of the cardinality objection is that empirical substructures always have infinite domains, but this is not necessarily so. To follow van Fraassen and allow an empirical embedding of both finite and infinite appearances, theories only need both finite and infinite empirical substructures. This is easily achieved, since for any model $\mathfrak{T}_{n}$ of a theory, the set of empirical substructures $\mathbf{E}_{n}$ can be closed under the substructure relation, that is, if $\mathfrak{E} \in \mathbf{E}_{n}$ and $\mathfrak{E}^{\prime} \subseteq \mathfrak{E}$, then $\mathfrak{E}^{\prime} \in \mathrm{E}_{n}$, so that with every empirical substructure $\mathfrak{E}$, all of $\mathfrak{E}$ 's finite substructures are empirical substructures as well. In other words, the cardinality objection fails because it assumes a feature of all empirical substructures (i. e., infinite domains) not entailed by van Fraassen's position. This response to

14. I do not endorse van Fraassen's argument. For my point it is only important that the argument presumes that theories can be described as classes of model theoretic structures. In fact, I think that van Fraassen's position is wrong and the exchange between Worrall, Friedman, and van Fraassen is a red herring, since, first, the paradigmatic syntactic approach of the logical empiricists explicitly assumes higher order logic (Lutz 2012, \$2), and second, empirical adequacy can provably be expressed in higher order logic (Lutz 2014a).

15. Incidentally, since van Fraassen relies on model theory, his alleged freedom from being "yolked to a particular syntax" can only amount to the freedom from a particular set of nonlogical constants in the object language as it is found in the definitions by Bell and Slomson (1974). For even in those definitions, the use of a model theoretic structure always fixes a particular syntax up to the choice of a set of non-logical constants (cf. Lutz 2014b, $\$ 3$ ). 
the cardinality objections is restricted only by the problems stemming from the technical aspects of substructures discussed above (it is impossible, for instance, if the theory involves a successor function on its whole domain). Hence a solution to these problems will also provide a response to the cardinality objection.

This, then, leaves the problems stemming from the use of substructures, the lack of a notion of epistemic appearances and of approximate appearances, and the lack of a notion of information gain about the appearances. These problems can be solved by further developing empirical adequacy, which, it turns out, can be done by looking at its origins.

\section{Approximation sets}

Van Fraassen $(1980,64 ; 1989,227)$ traces his notion of empirical substructure back to a monograph by Przełęcki (1969) and other works on the application of model- and set theory in the philosophy of science, only noting that "some of these formulations were still more language-oriented than [he] liked" (1989, 227). However, the connection between Przelęcki's monograph and constructive empiricism is not obvious, to put it mildly: Van Fraassen $(1980, \$ 3.6)$ famously declared that the (so-called) received view on scientific theories, as developed by Carnap, Hempel, and others within logical positivism, is in principle unable to describe the correct relation between theory and phenomena. Przełęcki (1975, 284), on the other hand, thought of himself as "positivistically-minded" and of the monograph as an introduction to the received view (Przelęcki 1974b, 402). ${ }^{16}$ Przełęcki’s discussion differs from previous expositions (e. g. Carnap 1939, \$24) mainly in that he explicitly develops the model theoretic implications of the received view and discusses approximations. It is this latter discussion, and its elaboration that Przełęcki (1976) published after Fine (1975, n. 13) had developed a similar idea, that provide the relation to constructive empiricism. ${ }^{17}$

Starting from a discussion of vague terms, Przełęcki $(1976,378)$ suggests treating approximations with the help of sets of structures. The denotation of a relation symbol $R_{i}$ that is only approximately determined over some domain $A$ tripartitions the product domain $A^{m_{i}}$ into a set $R_{i}^{+}$of definite instances (the positive extension of $R_{i}$ ), a set $R_{i}^{-}$of definite non-instances (the negative extension), and a set of borderline cases of $R_{i}$, which I will call $R^{\circ}$ (the neutral extension). The denotation of a function symbol $F_{j}$ that is approximately determined over $A$ does not assign a single element $b \in A$ to an $n_{j}$-tuple $\left\langle a_{1}, \ldots, a_{n_{j}}\right\rangle \in A^{n_{j}}$, but rather a set $F_{j}^{+\circ}\left(a_{1}, \ldots, a_{n_{j}}\right)=B \subseteq A(375) \cdot{ }^{18} B$ can be seen as the set of possi-

16. It is hence fascinating to see Przełęcki's work cited as a precursor or even an elaboration of the semantic view (da Costa and French 1990, 249; Volpe 1995, 566), even though the semantic view, in which van Fraassen's notion of empirical adequacy is phrased, is usually considered to be diametrically opposed to the received, or "syntactic", view.

17. For a more thorough discussion of Przełęcki's formalism, see Lutz (2013).

18. This is a slight generalization of Przełęcki's account, who assumes that $B$ is an interval of 
ble values of the function named by $F_{j}$ for the arguments $a_{1}, \ldots, a_{n_{j}}$, and I will refer to the set $\left\{\left\langle a_{1}, \ldots, a_{n_{j}}, b\right\rangle: a_{1}, \ldots, a_{n_{j}} \in A, b \in F_{j}^{+o}\left(a_{1}, \ldots, a_{n_{j}}\right)\right\}$ as the nonnegative extension $F^{+\circ}$ of $F_{j} \cdot{ }^{19}$ Considering constant symbols 0 -place function symbols, this means that the denotation of a constant symbol $c_{k}$ that is approximate over $A$ is a set $c_{k}^{+o} \subseteq A$.

For a function symbol $F_{j}, F_{j}^{+\circ}$ may contain unintended functions. For example, unless $F_{j}$ has a positive extension over the whole domain, $F_{j}^{+\circ}$ contains discontinuous functions, which may go against the intended denotation of $F_{j}$. Przełęcki $(1976,376)$ therefore allows the denotation of a function symbol $F_{j}$ to be further determined by a set of "additional conditions" $W\left(F_{j}\right)$. Similarly to Przeęecki's additional conditions are what Fine $(1975,124)$ calls "penumbral connections", sentences that have to be true for all extensions of all terms. However, Fine assumes that these connections are given in the object language, not in the meta-language determining the denotations, and he does not restrict the penumbral connections to functions only. I will follow Przełęcki in assuming that the penumbral connections are given in the meta-language, but I will follow Fine in allowing penumbral connections for all terms. Specifically, I will assume that the penumbral connections determine a penumbral set $\mathbf{P}$ of structures.

The denotations of approximate terms over $A$ and the penumbral set $\mathbf{P}$ immediately lead to a set of of structures:

Definition 5. Let the terms $\left\{R_{i}, F_{j}, c_{k}\right\}_{i \in I, j \in J, k \in K}$ be approximate over domain $A$ with positive, negative, and non-negative extensions $\left\{R_{i}^{+}, R_{i}^{-}, F_{j}^{+\circ}\right.$, $\left.c_{k}^{+\circ}\right\}_{i \in I, j \in J, k \in K}$, and penumbral set $\mathbf{P}$. Then the terms' approximation set $\mathbf{M}$ for $A$ contains all and only structures $\mathfrak{M} \in \mathbf{P}$ for which

$$
\begin{gathered}
M=A, \\
R_{i}^{+} \subseteq R_{i}^{\mathfrak{M}} \subseteq A^{m_{i}}-R_{i}^{-} \text {for all } i \in I, \\
F_{j}^{\mathfrak{M}} \subseteq F_{j}^{+\circ} \text { for all } j \in J, \text { and } \\
c_{k}^{\mathfrak{M}} \in c_{k}^{+o} \text { for all } k \in K .
\end{gathered}
$$

In the following, I will assume that the approximation set for the terms and the penumbral set is never empty, that is, the penumbral connections are not in conflict with the positive, negative, and non-negative extensions of the terms. Furthermore, I will assume that the penumbral set is only used to exclude those structures from approximation sets that cannot be excluded with the help of positive, negative, and non-negative extensions. More precisely, I will assume that for any approximation set $\mathbf{M}$ over domain $A$ for terms with $\left\{R_{i}^{+}, R_{i}^{-}, F_{j}^{+\circ}\right.$,

reals, which would therefore have to be in $A$.

19. $F_{j}^{+\circ}$ is the union of an approximate relation symbol's positive and neutral extensions. 
$\left.c_{k}^{+\circ}\right\}_{i \in I, j \in J, k \in K}$ and $\mathbf{P}$,

$$
\begin{aligned}
R_{i}^{+} & =\bigcap\left\{R_{i}^{\mathfrak{M}}: \mathfrak{M} \in \mathbf{M}\right\}, \\
R_{i}^{-} & =\bigcap\left\{A^{m_{i}+1}-R_{i}^{\mathfrak{M}}: \mathfrak{M} \in \mathbf{M}\right\}, \\
F_{j}^{+o} & =\bigcup\left\{F_{j}^{\mathfrak{M}}: \mathfrak{M} \in \mathbf{M}\right\}, \text { and } \\
c_{k}^{+o} & =\bigcup\left\{c_{k}^{\mathfrak{M}}: \mathfrak{M} \in \mathbf{M}\right\}
\end{aligned}
$$

for all $i \in I, j \in J, k \in K$.

An approximation set $\mathbf{M}$ represents those structures that approximate the right structure (or set of structures). The right structure will thus be in $\mathbf{M}$, together with other structures that are close enough to the right one. Note that approximation sets are extremely general, so that, formally, not much is assumed about the nature of approximation. Approximate truth is then truth in a structure approximating the right structure or set of structures (Przełęcki 1976, 378):

Definition 6. Let $\mathbf{M}$ be an approximation set. A set of sentences is approximately true if and only if in at least one $\mathfrak{M} \in \mathbf{M}$ all its elements are true. A sentence is approximately true if and only if its singleton set is approximately true.

The definition of approximate truth for sets of sentences avoids inconsistent approximately true sets. In this way, a finite set of sentences is approximately true if and only if the conjunction of its elements is approximately true. Of course, two incompatible sentences can both be approximately true, so that there are sets of approximately true sentences that are inconsistent and that hence are no approximately true sets of sentences. In other words, approximate truth is not preserved under conjunction.

Przełęcki's definition 6 determines approximate truth only for sets of sentences $H$, which illustrates van Fraassen's remark that some of his inspirations were more language-oriented than he liked. But this language-orientation can be avoided with a slight generalization. It follows from definition 6 that $H$ is approximately true if and only if a model of $H$ is in $\mathbf{M}$. Since the class $\mathbf{E}$ of models of $H$ is closed under isomorphism, this means that $H$ is approximately true if and only if some $\mathfrak{E} \in \mathbf{E}$ is isomorphic to a structure in $\mathbf{M}$. And this conception of truth directly generalizes van Fraassen's conception of truth as isomorphism: Instead of demanding that some $\mathfrak{E} \in \mathbf{E}$ be isomorphic to the one right structure, one demands that some $\mathbb{E} \in \mathbf{E}$ be isomorphic to one of those structures in $\mathbf{M}$ that approximate the right one:

Definition 7. Let $\mathbf{M}$ be an approximation set. A class of structures $\mathbf{E}$ is approximately true in $\mathbf{M}$ if and only if for some $\mathfrak{E} \in \mathbf{E}$ and some $\mathfrak{M} \in \mathbf{M}, \mathfrak{E} \cong \mathfrak{M}$.

The connection between Przełęcki's formalism and constructive empiricism is now given by Przełęcki's treatment of unobservable objects and a generalization of his formalism by Przełęcki himself. Przełęcki $(1969, \$ 4)$ assumes that 
there is a fixed set of objects, some empirically determined to be observable, some empirically determined to be unobservable, and that all unobservable objects must be in the in the neutral or non-negative extensions of all terms. In other words, the approximation set $\mathbf{M}$ for the terms of a theory can be determined by, first, determining the approximation set $\mathbf{O}$ over the set of observable objects, and then including in $\mathbf{M}$ all extensions of members of $\mathbf{O}$ to all objects. Since $\mathfrak{M}$ is by definition an extension of $\mathfrak{O}$ if and only if $\mathfrak{M}$ is a substructure of $\mathfrak{O}$, van Fraassen's tracing back his use of empirical substructures to Przełęcki (1969) becomes understandable. Since Przełęcki assumes a fixed set of objects, however, his formalism presumes that one has some kind of direct access to unobservable objects (Przełęcki 1974b, 405), which van Fraassen $(1989, \$ 3$ ) very much denies.

\section{Generalizations}

Definition 7 achieves the language independence that van Fraassen demands, and so Przełęcki's formalism can now be used to generalize empirical adequacy as long as any assumption about directly accessible unobservable objects is avoided. This has the immediate advantage of relying only on conceptual assumptions that van Fraassen could accept, and in fact was inspired by. To be adequate, however, the generalizations of empirical adequacy to epistemic and approximate empirical adequacy will have to fulfill some further conditions.

The first condition is that epistemic empirical adequacy indeed has to be a generalization of empirical adequacy. That is, whenever everything that is possible to know about the appearances is known, epistemic empirical adequacy should be equivalent to empirical adequacy. When not everything is known, epistemic empirical adequacy should be properly weaker than empirical adequacy. This condition of adequacy also suggests two more: First, the generalization should, like definition 3 of empirical adequacy, be metaphysically neutral, while allowing a strict empiricism. Second, it should not confer a distinguished status upon human perception, and thus be as neutral with respect to van Fraassen's epistemological positions as definition 3 .

Further, the example of the interference phenomenon suggests that it is very fruitful to develop approximate empirical adequacy as a special case of epistemic empirical adequacy. Specifically, an approximate value of a quantity should be treated formally as a lack of knowledge about the precise value of the quantity. In this way, a theory $\left\langle\mathfrak{T}_{n}, \mathbf{E}_{n}\right\rangle$ that was epistemically empirically adequate given the resolution of the best measurements can still be valued for its approximate empirical adequacy, even if upon more precise measurements, $\left\langle\mathfrak{T}_{n}, \mathbf{E}_{n}\right\rangle$ ceases to be epistemically empirically adequate. Given the two concepts' relation, any conditions of adequacy for epistemic empirical adequacy also hold for approximate empirical adequacy.

For practical purposes, results about the generalizations of empirical adequacy should transfer sensibly from finite to infinite domains. Specifically, the 
generalizations of empirical adequacy for appearances with an infinite domain should be limiting cases of the generalizations for appearances with a finite domain. Otherwise it would, for example, be impossible to infer anything about surface models from data models, and thus it would specifically be impossible to argue for the empirical adequacy of a theory for appearances of a continuum with the help of any finite number of measurements.

\subsection{Empirical relativized reducts}

In van Fraassen's definition 1 of a theory, the components of a theory that are connected to the appearances are given by empirical substructures. This leads to a variety of problems, since all the terms that occur in the theory also have to occur in the substructures. The problem suggests the following generalization of definition 1:

Definition 8. A theory $\left\langle\mathfrak{T}_{n}, \mathbf{E}_{n}\right\rangle$ contains a family of structures (the models of the theory) and for each structure $\mathfrak{T}_{n}=\left\langle T_{n}, P_{i}^{\mathfrak{T}_{n}}, F_{j}^{\mathfrak{T}_{n}}, c_{k}^{\mathfrak{T}_{n}}\right\rangle_{i \in I_{n}, j \in J_{n}, k \in K_{n}}$ a set $\mathbf{E}_{n}$ of empirical relativized reducts, such that for each $\mathfrak{E} \in \mathbf{E}_{n}$, there is a set $\mathscr{A}_{\mathfrak{E}}$ of terms such that $\left.\mathfrak{E} \subseteq \mathfrak{T}_{n}\right|_{\mathscr{A}}$. With each structure, a theory also contains every isomorphic model and its corresponding empirical substructures.

The reduct $\left.\mathfrak{T}_{n}\right|_{\mathscr{A}_{\mathfrak{E}}}$ of $\mathfrak{T}_{n}$ to $\mathscr{A}_{\mathfrak{E}}$ is the structure that differs from $\mathfrak{T}_{n}$ only in that it provides no interpretation of those terms not in $\mathscr{A}_{\mathfrak{E}}$ (Hodges 1993, 9). This means that every empirical relativized reduct $\mathfrak{E} \in \mathbf{E}_{n}$ is a relativized reduct in the sense of model theory. ${ }^{20}$ Przelęcki (1969, ch.5-6) builds much of his formalism on reducts, and Suárez $(2005,38)$ implicitly uses relativized reducts instead of substructures in his discussion of empirical adequacy. ${ }^{21}$ Muller $(1997$, n. 2$)$ is explicit about his "non-standard" interpretation of 'substructure' as 'substructure of a contraction', where 'contraction' means 'reduct'. ${ }^{22}$ It is telling that the discussions of both Suárez and Muller are applications of semantic formalisms to specific theories, and both authors found the concept of a substructure (which in the abstract seems very appealing) too confining. With its use of relativized reducts, definition 8 now solves the problems connected with the use of substructures: There can be unobservable constants, and functions from values of measurement results to other mathematical entities, since the constants' and functions' symbols may not be in the vocabulary $\mathscr{A}_{\mathfrak{E}}$.

Although Przełęcki uses reducts to capture the received view, the use of relativized reducts is not a relapse into Carnap's or Hempel's conception of a theory, since the notion of a substructure has not been abandoned. What is more, definition 8 of a theory contains definition 1 as a special case, namely when for each

20. Hodges $(1993,202-203)$ defines relativized reducts as those substructures of a reduct that have the extension of some one place predicate as their domain. I use a slight generalization.

21. Since this again weakens his notion of empirical adequacy, his overall argument remains unaffected (see n. 11).

22. Of course, Muller does not claim to be using van Fraassen's notion of empirical adequacy. 
$\mathfrak{E} \in \mathbf{E}_{n}, \mathscr{A}_{\mathfrak{E}}$ contains all symbols of $\mathfrak{T}_{n}$, so that $\left.\mathfrak{T}_{n}\right|_{\mathscr{A}_{\mathfrak{E}}}=\mathfrak{T}_{n}$ and thus $\mathfrak{E} \subseteq \mathfrak{T}_{n}$. Then a theory can be formalized as before.

Furthermore, one could even avoid the use of relativized reducts in the formalization of a theory by first treating functions and constants as special kinds of relations, thereby reformulating the theory to contain only relations. For each model $\mathfrak{T}_{n}$ of the theory, define then a new model $\mathfrak{T}_{n}^{\prime}$ with at least one object $t \in T_{n}^{\prime}-\bigcup\left\{E: \mathfrak{E} \in \mathbf{E}_{n}\right\}$ such that $\left.\left.\mathfrak{T}_{n}\right|_{\mathscr{A}_{\mathbb{E}}} \subseteq \mathfrak{T}_{n}^{\prime}\right|_{\mathscr{A}_{\mathbb{E}}}$ and for each $m_{i}$-ary theoretical relation $R_{i}^{\mathfrak{T}_{n}}, \mathfrak{T}_{n}^{\prime}$ contains an $m_{i}+1$-ary relation $\left\{\left\langle x_{1}, \ldots, x_{m_{i}}, t\right\rangle:\left\langle x_{1}, \ldots, x_{m_{i}}\right\rangle \in\right.$ $\left.R_{i}^{\mathfrak{T}_{n}}\right\}$. Then for every $\mathfrak{E} \in \mathbf{E}_{n}$, the substructure of $\mathfrak{T}_{n}^{\prime}$ with domain $E$ differs from $\mathfrak{E}$ only by empty relations, so that the empirical relativized reducts of the original theory can be considered the empirical substructures of the reformulated theory. ${ }^{23}$ Given the possibility of this reformulation, the use of relativized reducts cannot in principle be a problem. However, since reformulating theories exclusively in relation terms needlessly complicates their application (Hodges 1993, 2), and redefining some of these relations to always contain an unobservable object is not a paragon of simplicity either, the use of relativized reducts is preferable in the following.

Finally, one might object to definition 8 because through its reference to a set of symbols, it appears much more language dependent than van Fraassen's definition 1. This appearance is misleading, as discussed at the beginning of $\mathbb{S}_{3}$ : Depending on the notation, either definition 1 implicitly contains a reference to a set of symbols, or definition 8 can be reformulated without reference to a set of symbols, using an index set instead (cf. Lutz 2014b, $\mathbb{\$} 3$ ). Muller $(1997$, n. 2) also provides a definition of reduct in the standard notation of the semantic view.

\subsection{Epistemic empirical adequacy}

Generalizing substructures to relativized reducts in the definition of a theory has solved the problems connected with empirical adequacy's exclusive reliance on substructures. Generalizing structures to approximation sets of structures will solve empirical adequacy's problems with incomplete knowledge. Indeed, it will be useful to generalize approximation sets even further and allow any kind of set of structures, since it is doubtful that every lack of knowledge can be expressed as an approximation (one may, for instance, be ignorant about the number of objects in the domain). This generalization allows capturing lack of knowledge in the following way: A description of an appearance by a single structure is maximally informative within the conceptual framework of the theory, simply because the structure of the appearance is completely determined. A description of an appearance by two structures is less informative, since the appearance could have either of the two structures. In general, the more structures are used to describe an appearance, the less information the description provides. Hence it is possible to express the lack of knowledge about a specific appearance by a set

23. I thank Albert Visser for this point. 
of structures: For all the scientist knows, the actual appearance could have any of these structures. A singleton set of structures expresses certainty about the structure of the appearance. Definition 2 thus becomes

Definition 9. Epistemic appearances are given by a set $\mathbf{A}$ of sets of structures. A set $\mathbf{Q} \in \mathbf{A}$ is an epistemic appearance.

Note that, like definition 2, definition 9 allows the complete lack of knowledge about some appearances: The set A may, for instance, contain only a single set of structures, even though there are many more appearances that could be described, however imprecisely.

Definition 7 of approximate truth can now also be generalized: A class of structures $\mathbf{E}$ is then epistemically true in $\mathbf{Q}$, that is, true to the extent of one's knowledge, if for some $\mathfrak{E} \in \mathbf{E}$ and some $\mathfrak{A} \in \mathbf{Q}, \mathfrak{E} \cong \mathfrak{A}$. Informally, the empirical adequacy of a theory is the truth of the theory's claims about the phenomena. The pertinent generalization in this case would be that the theory's claims about the phenomena are epistemically true. Since what a theory claims about the phenomena is given by its empirical substructures, this leads directly to

Definition 10. Given the epistemic appearances $\mathbf{A}$, a theory $\left\langle\mathfrak{T}_{n}, \mathbf{E}_{n}\right\rangle$ is epistemically empirically adequate for $\mathbf{A}$ if and only if there is some $n \in N$ such that for every $\mathbf{Q} \in \mathbf{A}$, there are an $\mathfrak{A} \in \mathbf{Q}$ and an $\mathfrak{E} \in \mathbf{E}_{n}$ with $\mathfrak{E} \cong \mathfrak{A}$.

As noted, definition 3 expresses empirical adequacy if and only if $\mathbf{A}$ is assumed to contain all appearances, and in this sense, it already allows for a modified notion of empirical adequacy that takes a specific kind of lack of knowledge into account: Some appearances may simply not be described at all. Definition 10 adds a new kind of lack of knowledge, since even those appearances that are described may still be described incompletely.

Example. It is now possible to investigate under what circumstances the ray theory of light is epistemically empirically adequate given the interference phenomenon. Let the measurement set-up be such that the scientist's eyes have the effective spatial resolution $p>p_{\min }$ and intensity resolution $q>q_{\min }$. If the intensity pattern is measured, giving a finite set of pairs of values

$$
\left\{\left\langle x_{1}, \bar{\psi}_{p}^{q}\left(x_{1}\right)\right\rangle, \ldots,\left\langle x_{s}, \bar{\psi}_{p}^{q}\left(x_{s}\right)\right\rangle\right\}
$$

then the effective resolutions of the scientist's eyes restrict the possible actual values of the appearance $\bar{\psi}_{p_{\min }}^{q_{\min }}$. The measurements thus result in the set

$$
\{\langle\mathbb{R}, \varphi\rangle: \varphi \in \Phi\}
$$

of structures, where all functions $\varphi \in \Phi$ have to be such that

$$
\varphi: \mathbb{R} \longrightarrow \wp \mathbb{R}^{\geq 0}, x \mapsto\left[\chi(x)-\frac{q_{\min }}{2}, \chi(x)+\frac{q_{\min }}{2}\right] \cap \mathbb{R}^{\geq 0}
$$


for some $\chi: \mathbb{R} \longrightarrow \mathbb{R}$ and $\varphi\left(x_{i}\right) \subseteq \bar{\psi}_{p}^{q}\left(x_{i}\right)$ for all $1 \leq i \leq s$. One of these functions then is the actual graph $\bar{\psi}_{p_{\min }}^{q_{\min }}$ of the intensity, but for all the scientist knows, it could be any of them. Note that the epistemic appearances are already given as a set of structures with infinite domains, thereby avoiding the cardinality objection from the start.

For the ray theory to be epistemically empirically adequate, the measured intensity ranges $\bar{\psi}_{p}^{q}\left(x_{i}\right)$ must be supersets of the ranges $\overline{\psi_{\text {ray }}^{\text {ray }}} q_{\text {min }}\left(x_{i}\right)=$ $\left[I-q_{\min } / 2, I+q_{\min } / 2\right] \cap \mathbb{R}^{\geq 0}$. This is the case if $\bar{\psi}_{p}$ never deviates from $\overline{\psi^{\text {ray }}}{ }_{\text {min }}$ more than $\left(q-q_{\min }\right) / 2$. In terms of the deviation factor (9), this means that $2 I|D(p / \lambda)| \leq q-q_{\min }$ for all $\lambda$ (since in this case $p$ is assumed fixed). Since $D(x)$ is enclosed between $\pm 1 /(2 \pi x)$, this is always the case if $\lambda I \leq \pi p\left(q-q_{\min }\right){ }^{24} \star$

The connection between epistemic empirical adequacy and empirical adequacy can be formulated with the help of

Definition 11. Given epistemic appearances $\mathbf{A}, \mathbf{A}^{\prime}$ are epistemically possible appearances if and only if $\mathbf{A}^{\prime}=\{e(\mathbf{Q}): \mathbf{Q} \in \mathbf{A}\}$, where $e$ is any function from $\mathbf{A}$ to $\bigcup \mathbf{A}$ with $e(\mathbf{Q}) \in \mathbf{Q}$.

$e$ is a choice function, picking out one element $\mathfrak{A}=e(\mathbf{Q})$ from each $\mathbf{Q}$. This is the formal expression of the idea that for each set of structures describing a single appearance, each of the structures could be the correct description of the appearance. A theory $\left\langle\mathfrak{T}_{n}, \mathbf{E}_{n}\right\rangle$ is then epistemically empirically adequate for epistemic appearances $\mathbf{A}$ if and only if there are epistemically possible appearances $\mathbf{A}^{\prime}$ such that $\left\langle\mathfrak{T}_{n}, \mathbf{E}_{n}\right\rangle$ is empirically adequate for $\mathbf{A}^{\prime}$ (claim 3 ). Thus the epistemic appearances can indeed be considered the set of epistemically possible appearances.

It is now possible to show that the conception of epistemic adequacy suggested here fulfills the conditions of adequacy. For one, whenever the appearances are completely known (without any uncertainty as expressed by a multiplicity of structures), epistemic empirical adequacy is equivalent to empirical adequacy. More precisely, if $\mathbf{A}$ are the appearances and $\mathbf{A}^{\prime}=\{\{\mathfrak{A}\}: \mathfrak{A} \in \mathbf{A}\}$ are epistemic appearances, then $\left\langle\mathfrak{T}_{n}, \mathbf{E}_{n}\right\rangle$ is empirically adequate for $\mathbf{A}$ if and only if $\left\langle\mathfrak{T}_{n}, \mathbf{E}_{n}\right\rangle$ is epistemically empirically adequate for $\mathbf{A}^{\prime}$ (claim 4 ). Thus, as required, definition 10 generalizes van Fraassen's definition 3 of empirical adequacy.

Definitions 9 and 10 rely only on concepts that are defined both for finite and infinite domains, and that are known to behave well in the transition from one to the other. Since furthermore the definitions themselves do not refer to the cardinality of the domains, it can be expected that they, too, behave well in the transition from finite to infinite domains. The definitions also do not add any metaphysical assumptions. The only additional assumption, that lack of knowledge can be represented as multiplicity, is epistemic. Finally, nothing in

24. This holds for $p / \lambda<1 /(2 \pi)$ (see n. 3 ). 
definitions 9 and 10 requires that the appearances describe direct observations. They could also be determined through measurements that are, for the purposes at hand, treated as uncontroversial. ${ }^{25}$

\subsection{Approximate empirical adequacy}

As demanded by the conditions of adequacy, one can now define approximation as a special case of lack of knowledge:

Definition 12. Approximate appearances are given by epistemic appearances $\mathbf{A}$ where each $\mathbf{Q} \in \mathbf{A}$ is an approximation set.

Accordingly, approximate empirical adequacy is a special case of epistemic empirical adequacy:

Definition 13. A theory $\left\langle\mathfrak{T}_{n}, \mathbf{E}_{n}\right\rangle$ is approximately empirically adequate for approximate appearances $\mathbf{A}$ if and only if $\left\langle\mathfrak{T}_{n}, \mathbf{E}_{n}\right\rangle$ is epistemically empirically adequate for $\mathbf{A}$.

It follows from definitions 10 and 13 that $\left\langle\mathfrak{T}_{n}, \mathbf{E}_{n}\right\rangle$ is approximately empirically adequate given $\mathbf{A}$ if and only if there is an $n \in N$ such that for every approximate appearance $\mathbf{Q} \in \mathbf{A}$ there is an empirical substructure $\mathbb{E} \in \mathbf{E}_{n}$ that is isomorphic to some member of $\mathbf{Q}$. Definition 13 thus provides an explication of van Fraassen's unexplicated notion of "approximate embedding" (van Fraassen 1989 , 366, n. 5). ${ }^{26}$ Given definition 7 , one can also say that a theory is approximately empirically adequate if and only if what it says about the phenomena is approximately true.

Since singleton sets of structures are approximation sets, and since by claim 4 epistemic empirical adequacy given singleton epistemic appearances corresponds to empirical adequacy, it follows that definition 13 generalizes van Fraassen's definition 3 of empirical adequacy. In general, the connection between approximate empirical adequacy and empirical adequacy is the same as that between epistemic empirical adequacy and empirical adequacy (and thus given by definition 11 and claim 3).

Example. The interference phenomenon can be treated in terms of approximate empirical adequacy as well. Indeed, the conditions (20) of the epistemic appearance (19) are already such that the epistemic appearance is an approximation set. For the non-negative extension of the function $\varphi$ is given by

$$
\begin{aligned}
\varphi^{+\circ}=\left\{\left\langle x_{i},\left[y_{i}-\frac{q_{\min }}{2}, y_{i}+\frac{q_{\min }}{2}\right] \cap \mathbb{R}^{\geq 0}\right\rangle:\right. & \\
& {\left.\left[y_{i}-\frac{q_{\min }}{2}, y_{i}+\frac{q_{\min }}{2}\right] \cap \mathbb{R}^{\geq 0} \subseteq \bar{\psi}_{p}^{q}\left(x_{i}\right), 1 \leq i \leq s\right\} . }
\end{aligned}
$$

25. The generalization even allows treating a non-blurred interference pattern as appearance, and the blurred interference pattern as an epistemic appearance. Of course, this would go against the distinguished status that constructive empiricism confers upon human perception.

26. Although I would call it 'approximate empirical embedding'. 
Hence, for the ray theory to be approximately empirically adequate, the measured intensity ranges must again be supersets of $\left[I-q_{\min } / 2, I+q_{\min } / 2\right] \cap \mathbb{R} \geq 0$.

However, even if at some point $p=p_{\min }$ and $q=q_{\min }$, the ray theory can still be approximately true in the sense that, for some resolutions $p_{0}$ and $q_{0}$, $\underset{p_{\text {min }}}{\overline{\text { ray }}} q_{\min }$ always stays within the range of the blurred intensity $\bar{\psi}_{p_{0}}^{q_{0}}$. Thus the ray theory stays approximately empirically adequate. If one assumes $p_{\min }=0$ (or rather: if one takes the convolution function to be a delta distribution, $b(x)=$ $\delta(x)$ ) and further assumes $q_{\min }=0$, one has effectively eliminated the auxiliary theory about the scientist's eyes from the preceding analysis. The analysis then only pertains to $\overline{\psi^{\text {ray }}}$ and $\bar{\psi}$, and that $\overline{\psi^{\text {ray }}}(x) \in \bar{\psi}_{p_{0}}^{q_{0}}(x)$ expresses simply that the ray theory approximates the wave theory, in the specific contextual sense of 'approximation' given by $\bar{\psi}_{p_{0}}^{q_{0}}$. Note that contrary to claims in some discussions of the relation between wave and ray theory of light (e. g., Batterman 2002, \$6.2), there is no need to take any singular limits into account for this result.

\subsection{Hierarchies of empirical adequacy}

With epistemic and approximate empirical adequacy defined relative to epistemic and approximate appearances, one can now formalize the development of the appearances, specifically, the increase of knowledge about the phenomena. To this effect, I suggest

Definition 14. A bierarchy of epistemic appearances $\left\langle\left\{\mathbf{A}_{l}\right\}_{l \in L}, C\right\rangle$ contains an indexed set $\left\{\mathbf{A}_{l}\right\}_{l \in L}$ of epistemic appearances with an ordered index set $L$ and a set $C$ containing for any $\mathbf{A}_{l}, \mathbf{A}_{m}, l, m \in L, l \leq m$ exactly one injection $b: \mathbf{A}_{l} \longrightarrow$ $\mathbf{A}_{m}$ such that for all $\mathbf{Q} \in \mathbf{A}_{l}, b(\mathbf{Q}) \subseteq \mathbf{Q}$.

Additionally, for any three injections $b, c, d \in C$ with $b: \mathbf{A}_{l} \longrightarrow \mathbf{A}_{m}, c$ : $\mathbf{A}_{m} \longrightarrow \mathbf{A}_{n}$, and $d: \mathbf{A}_{l} \longrightarrow \mathbf{A}_{n}, d=c \circ b$.

To see the point of the definition, consider for now only the first part. A hierarchy of epistemic appearances allows the growth of knowledge in two respects. First, since each $b \in C$ only has to be an injection, $\mathbf{A}_{m}$ can contain more epistemic appearances than $\mathbf{A}_{l}$. Hence an epistemic hierarchy can describe the accumulation of measurements of new phenomena. Second, since $b(\mathbf{Q}) \subseteq \mathbf{Q}$, the knowledge about specific appearances can grow as well, for the number of epistemically possible appearances can decrease. In this way, definition 14 captures the gain of knowledge from one level of the hierarchy to the next, or better, since the index set $L$ does not have to be countable, the gain of knowledge going from one level in the hierarchy (as indicated by $l \in L$ ) to any higher level. ${ }^{27}$

27. I thank Leszek Wroński for the suggestion to allow infinitely many levels. Note that the kind of hierarchy described here is very different from the hierarchy of structures described by Suppes (1962), which at least prima facie does not describe an increase in empirical information, but rather a successive change of structures given the same information. 
Because of the additional condition, $C$ determines for any appearance at any level in the hierarchy a unique line of ancestors and descendants. The effect of the condition is that it does not matter whether one makes first a step from $\mathbf{A}_{l}$ to $\mathbf{A}_{m}$ and then a step from $\mathbf{A}_{m}$ to $\mathbf{A}_{n}$ or directly one bigger step from $\mathbf{A}_{l}$ to $\mathbf{A}_{n}$. For the analysis of one's improvement of the appearances, this means that not every intermediate step in the improvement has to be explicitly included in one's analysis. The set $C$ also fulfills a second function: It determines which appearances at a level are improvements of which appearances at lower levels. Specifically, this means that $C$ determines which appearances from different levels describe the same phenomenon. ${ }^{28}$

Example. In the interference phenomenon, the measurement results (18) lead to the epistemic appearance (20), which is determined by the spatial resolution $p$ and the intensity resolution $q$. For intensity resolutions $q^{\prime} \leq q$, the epistemic appearance is a subset of that for $q$, so that an increase in resolution (i. e., a decrease of $q$ ) leads naturally to a hierarchy of epistemic appearances. A decrease of $p$ does not lead to such a hierarchy. Rather, changing $p$ leads to different hierarchies, each depending on $q$ : For $p=n \lambda / 2$ and for $p \rightarrow \infty$, the deviation factor $D(p / \lambda)=0$, so that $\bar{\psi}_{p}^{q}=\overline{\psi^{\text {ray }}} q_{\text {min }}=[I-q / 2, I+q / 2] \cap \mathbb{R}^{\geq 0}$. Other values $0 \leq p<\lambda$ lead to sinusoidal patterns.

A theory that is found out not to be epistemically empirically adequate at some point should not become epistemically empirically adequate when the knowledge about the phenomena increases, and vice versa, a theory epistemically empirically adequate at one point should also be epistemically empirically adequate when less is known about the phenomena. This is the case for hierarchies of epistemic appearances (claim 5).

As a special case of definition 14 , there is

Definition 15. A restricted bierarchy of epistemic appearances is a hierarchy of epistemic appearance in which the injections between epistemic appearances are bijections.

A restricted hierarchy captures the idea that the increase of knowledge may be restricted to describing specific phenomena more precisely, rather than considering new phenomena. A restricted hierarchy provides a much tighter control over the appearances. This will become clear below.

Relative to a hierarchy of epistemic appearances, it is now possible to define what it means for one theory to be more epistemically empirically adequate

28. Note that one could also express successive improvements in the precision of measurements through measuring devices in terms of hierarchies of epistemic appearances. Of course, this would again go against the distinguished status that constructive empiricism confers upon human perception. 
than another. As the discussion of definition 2 has made clear, such a comparison based on appearances is only possible between theories that have the same conceptual framework. ${ }^{29}$

Definition 16. Given a (restricted) hierarchy of epistemic appearances $\left\langle\left\{\mathbf{A}_{l}\right\}_{l \in L}\right.$, $C\rangle$, theory $\left\langle\mathfrak{T}_{n}, \mathbf{E}_{n}\right\rangle$ is at least as (restrictedly) epistemically empirically adequate for $\left\langle\left\{\mathbf{A}_{l}\right\}_{l \in L}, C\right\rangle$ as theory $\left\langle\mathfrak{T}_{s}, \mathbf{E}_{s}\right\rangle$ if and only if for any $l \in L$ it holds that $\left\langle\mathfrak{T}_{s}, \mathbf{E}_{s}\right\rangle$ is epistemically empirically adequate for $\mathbf{A}_{l}$ only if $\left\langle\mathfrak{T}_{n}, \mathbf{E}_{n}\right\rangle$ is epistemically empirically adequate for $\mathbf{A}_{l}$.

Since an epistemically empirically inadequate theory can never become epistemically empirically adequate by an increase in knowledge about the appearances (claim 5), definition 16 entails that if theory $\left\langle\mathfrak{T}_{n}, \mathbf{E}_{n}\right\rangle$ is at least as epistemically empirically adequate as theory $\left\langle\mathfrak{T}_{s}, \mathbf{E}_{s}\right\rangle$ and $\left\langle\mathfrak{T}_{n}, \mathbf{E}_{n}\right\rangle$ ceases to be empirically adequate due to knowledge gain, so does $\left\langle\mathfrak{T}_{s}, \mathbf{E}_{s}\right\rangle$.

The definition of a hierarchy for approximations and the corresponding definition of comparative approximate empirical adequacy are straightforward:

Definition 17. A (restricted) hierarchy of approximate appearances is a (restricted) hierarchy of epistemic appearances in which all members are approximation sets.

Definition 18. Given a (restricted) hierarchy of approximate appearances $\left\langle\left\{\mathbf{A}_{l}\right\}_{l \in L}, C\right\rangle$, theory $\left\langle\mathfrak{T}_{n}, \mathbf{E}_{n}\right\rangle$ is at least as (restrictedly) approximately empirically adequate for $\left\langle\left\{\mathbf{A}_{l}\right\}_{l \in L}, C\right\rangle$ as theory $\left\langle\mathfrak{T}_{s}, \mathbf{E}_{s}\right\rangle$ if and only if $\left\langle\mathfrak{T}_{n}, \mathbf{E}_{n}\right\rangle$ is at least as (restrictedly) epistemically empirically adequate for $\left\langle\left\{\mathbf{A}_{l}\right\}_{l \in L}, C\right\rangle$ as theory $\left\langle\mathfrak{T}_{s}, \mathbf{E}_{s}\right\rangle$.

The hierarchies of epistemic and approximate appearances allow for specific routes of the increase of knowledge about the phenomena, that is, different series of experiments will lead to different hierarchies. In principle the formalism does not even need to track any real or imagined series of experiments. It just has to track some kind of improvement. Since the comparative notions of approximate empirical adequacy are defined via hierarchies, the formalism suggested here can therefore capture in a very general way the idea that the quality of an approximation can depend on the context (which can determine the approximate hierarchies). This conception of comparative approximation does not render the concept completely arbitrary or exclusively dependent on the context, however. In a (restricted) hierarchy of approximate appearances, the terms at each level of the hierarchy are better approximations (that is, are more precisely determined) than the terms at any lower level of the hierarchy (claim 6). The approximate hierarchy only determines how this increase in precision develops.

Example. Since in the interference phenomenon the epistemic appearance (19) already forms an approximation set, lowering $q$ leads not only to a hierarchy

29. In definitions and claims here and in the following, texts in brackets has to be either systematically included or omitted, thus leading to two different definitions and claims. 
of epistemic, but also of approximate appearances, which again is non-trivial for $p \neq n \lambda / 2, \infty$. And the hierarchy of approximate appearances resulting from lowering $q$ renders the wave theory of light at least as approximately empirically adequate as the ray theory of light. Again, the lowering of $p$ does not lead to an approximate hierarchy.

According to claims 3 and 5 , a theory that at one level in the hierarchy is not epistemically empirically adequate is not empirically adequate. But it is also of interest at which point of a hierarchy one can be sure that a theory is empirically adequate. Given the possibility that a new phenomenon renders a theory empirically inadequate, such a claim has to be restricted to restricted hierarchies. Roughly, once one has reached a level in a restricted hierarchy of epistemic or approximate appearances at which a theory is empirically adequate for all epistemically possible appearances, it will remain epistemically empirically adequate or, respectively, approximately empirically adequate under any further information gain. More precisely, $\left\langle\mathfrak{T}_{n}, \mathbf{E}_{n}\right\rangle$ is epistemically or approximately empirically adequate at all points of all restricted hierarchies of epistemic or, respectively, approximate appearances with the initial indexed set $\left\{\mathbf{A}_{l}\right\}_{l \leq m}$ if and only if $\left\langle\mathfrak{T}_{n}, \mathbf{E}_{n}\right\rangle$ is empirically adequate for all appearances that are epistemically possible given $\mathbf{A}_{m}$ (claim 7).

One may think that all these new concepts have taken the discussion far from van Fraassen's intentions, even if his formalism was modified only minimally and in line with one of his inspirations. But this is not so, for even the notion of epistemic hierarchies connects almost directly to one of van Fraassen's concepts. To see this, note first that one can compare the epistemic empirical adequacy of theories independently of specific hierarchies of epistemic appearances. Specifically, $\left\langle\mathfrak{T}_{n}, \mathbf{E}_{n}\right\rangle$ is at least as (restrictedly) epistemically empirically adequate as $\left\langle\mathfrak{T}_{s}, \mathbf{E}_{s}\right\rangle$ for any (restricted) hierarchy of epistemic appearances if and only if $\left\langle\mathfrak{T}_{n}, \mathbf{E}_{n}\right\rangle$ is empirically adequate for all appearances $\mathbf{A}$ for which $\left\langle\mathfrak{T}_{s}, \mathbf{E}_{s}\right\rangle$ is empirically adequate (claim 8). The universal generalization loses all the information that comes from specific hierarchies, that is, specific ways in which information about the phenomena is gained.

This coarse-graining now connects the preceding discussion directly with a definition by van Fraassen $(1980,67)$, who states: "If for every model $M$ of $T$ there is a model $M^{\prime}$ of $T^{\prime}$ such that all empirical substructures of $M$ are isomorphic to empirical substructures of $M^{\prime}$, then $T$ is empirically at least as strong as $T^{\prime \prime \prime}$. It now holds that for two theories $\left\langle\mathfrak{T}_{n}, \mathbf{E}_{n}\right\rangle$ and $\left\langle\mathfrak{T}_{s}, \mathbf{E}_{s}\right\rangle$, there is for every $n \in N$ an $s \in S$ such that all empirical substructures in $\mathbf{E}_{n}$ are isomorphic to empirical substructures in $\mathbf{E}_{s}$ if and only if $\left\langle\mathfrak{T}_{s}, \mathbf{E}_{s}\right\rangle$ is empirically adequate for all appearances $\mathbf{A}$ for which $\left\langle\mathfrak{T}_{n}, \mathbf{E}_{n}\right\rangle$ is empirically adequate (claim 9). Thus, by claims 7 and 9 , if $\left\langle\mathfrak{T}_{n}, \mathbf{E}_{n}\right\rangle$ is at least as (restrictedly) epistemically adequate as $\left\langle\mathfrak{T}_{s}, \mathbf{E}_{s}\right\rangle$ for any (restricted) hierarchy of epistemic appearances, then $\left\langle\mathfrak{T}_{s}, \mathbf{E}_{s}\right\rangle$ is empirically at least as strong as $\left\langle\mathfrak{T}_{n}, \mathbf{E}_{n}\right\rangle$. This result connects the concepts newly introduced in this article directly with van Fraassen's comparative notion 
of empirical strength.

The result also points to a weakness of van Fraassen's notion: Most theories will be incomparable in their empirical strength. As in the case of comparative epistemic or approximate empirical adequacy, two theories are incomparable if their or their auxiliary theories' conceptual frameworks differ. But furthermore, even if the two theories' appearances are given within the same framework, it will often happen that for each theory, there are structures that can be empirically embedded in one theory but not the other, so that the theories have incomparable empirical strength. In this respect, there is an analogy between empirical adequacy and empirical strength: Both are helpful concepts, but both are also too crude to capture many aspects of scientific research.

\section{Conclusion}

The use of approximation sets allows generalizations of empirical adequacy to incorporate lack of knowledge and approximation. That the generalizations allow an analysis of the example of the interference pattern shows that the generalizations are applicable to imprecise measurements. That the generalizations further allow a simple analysis of the growth of scientific knowledge and connect directly to van Fraassen's concept of empirical strength show that they are fruitful for research in the philosophy of science.

If the generalizations seem more complicated than one would expect from previous generalizations, this impression may result from the reliance of previous generalizations on idiosyncratic empirical adequacy (definition 4), which can lead to simplifications. Some generalizations also leave the possibility of multiple empirical substructures and appearances implicit (cf. Bueno 1997), which also simplifies matters considerably. If, on the other hand, the generalizations still seem too simplistic, especially in that they do not explicitly take statistical methods into account, this impression is correct. The concepts suggested here are first steps towards even more general relations between theories and appearances.

\section{A Proofs}

Claim 1. For some appearances A, some theories are idiosyncratically empirically adequate but not empirically adequate.

Proof. Let the appearances be given by the set of the two structures $\{\langle\{1,2\}$, $\{1,2\}\rangle,\langle\{3,4\},\{3\}\rangle\}$. Let the theory be given by the family with members $\mathfrak{T}_{1}=$ $\langle\{0,1,2\},,\{0,1,2\}\rangle$ and $\mathfrak{T}_{2}=\langle\{3,4,5\},\{3\}\rangle$ as well as the singleton sets of empirical substructures $\mathbf{E}_{1}=\{\langle\{1,2\},\{1,2\}\rangle\}$ and $\mathbf{E}_{2}=\{\langle\{3,4\},\{3\}\rangle\}$. Let all other models of the theory be isomorphic to $\mathfrak{T}_{1}$ or $\mathfrak{T}_{2}$ and have the corresponding empirical substructures. Then the theory is idiosyncratically empirically adequate 
by virtue of the identity mapping on each of the appearances' domains, but it is not empirically adequate.

Claim 2. For some appearances $\mathbf{A}$ and theory $\left\langle\mathfrak{T}_{n}, \mathbf{E}_{n}\right\rangle,\left\langle\mathfrak{T}_{n}, \mathbf{E}_{n}\right\rangle$ is empirically adequate given $\mathbf{A}$, but $\left\langle\mathfrak{T}_{n}, \mathbf{E}_{n}\right\rangle$ has no model such that all appearances in $\mathbf{A}$ are empirical substructures of that model.

Proof. Let the appearances be given by the set of the two structures $\{\langle\{a, b\}$, $\{a, b\}\rangle,\langle\{c, d\},\{c\}\rangle\}$, where $a, b, c$, and $d$ are distinct objects. Let the theory be given by the family with the member $\mathfrak{T}_{1}=\langle\{1,2,3\},\{1,2\}\rangle$ and the set of empirical substructures $\mathbf{E}_{1}=\{\langle\{1,2\},\{1,2\}\rangle,\langle\{2,3\},\{2\}\rangle\}$. Let all other models of the theory be isomorphic to $\mathfrak{T}_{1}$ and have the corresponding empirical substructures. Then the theory is empirically adequate, but every bijection from $\{1,2,3\}$-and thus every isomorphism for $\mathfrak{T}_{1}$-maps 2 , the object shared by the empirical substructures, to a single object. Since the domains of the appearances do not share an element, the appearances therefore can never be empirical substructures of the same model of the theory.

Claim 3. A theory $\left\langle\mathfrak{T}_{n}, \mathbf{E}_{n}\right\rangle$ is epistemically empirically adequate for epistemic appearances $\mathbf{A}$ if and only if there are epistemically possible appearances $\mathbf{A}^{\prime}$ such that $\left\langle\mathfrak{T}_{n}, \mathbf{E}_{n}\right\rangle$ is empirically adequate for $\mathbf{A}^{\prime}$.

Proof. ' $\Rightarrow$ ': $\left\langle\mathfrak{T}_{n}, \mathbf{E}_{n}\right\rangle$ is epistemically empirically adequate for $\mathbf{A}$ if and only if there is some $n \in N$ such that for every $\mathbf{Q} \in \mathbf{A}$, there are $\mathfrak{A} \in \mathbf{Q}$ and $\mathfrak{E} \in \mathbf{E}_{n}$ with $\mathfrak{E} \cong \mathfrak{A}$. For each $\mathbf{Q}$, choose $e(\mathbf{Q})=\mathfrak{A}$. Then there is some $n \in N$ such that for every $\mathfrak{A} \in \mathbf{A}^{\prime}$, there is an $\mathfrak{E} \in \mathbf{E}_{n}$ with $\mathfrak{E} \cong \mathfrak{A}$, so that $\left\langle\mathfrak{T}_{n}, \mathbf{E}_{n}\right\rangle$ is empirically adequate for $\mathbf{A}^{\prime}$.

' $\Leftarrow$ ': Similar.

Claim 4. Let $\mathbf{A}$ be appearances, and $\mathbf{A}^{\prime}=\{\{\mathfrak{A}\}: \mathfrak{A} \in \mathbf{A}\}$ be epistemic appearances. Then $\left\langle\mathfrak{T}_{n}, \mathbf{E}_{n}\right\rangle$ is empirically adequate for $\mathbf{A}$ if and only if $\left\langle\mathfrak{T}_{n}, \mathbf{E}_{n}\right\rangle$ is epistemically empirically adequate for $\mathbf{A}^{\prime}$.

Proof. Given the epistemic appearances $\mathbf{A}^{\prime}=\{\{\mathfrak{A}\}: \mathfrak{A} \in \mathbf{A}\}$, the only epistemically possible appearances are given by A. Claim 4 now follows from claim 3 .

Claim 5. Let $\left\langle\left\{\mathbf{A}_{l}\right\}_{l \in L}, C\right\rangle$ be a hierarchy of epistemic appearances. If theory $\left\langle\mathfrak{T}_{n}, \mathbf{E}_{n}\right\rangle$ is epistemically empirically adequate for $\mathbf{A}_{l}, l \in L$, then $\left\langle\mathfrak{T}_{n}, \mathbf{E}_{n}\right\rangle$ is epistemically empirically adequate for any $\mathbf{A}_{k}, k \in L, k \leq$ l. If theory $\left\langle\mathfrak{T}_{n}, \mathbf{E}_{n}\right\rangle$ is not epistemically empirically adequate for $\mathbf{A}_{l}, l \in L$, then $\left\langle\mathfrak{T}_{n}, \mathbf{E}_{n}\right\rangle$ is not epistemically empirically adequate for any $\mathbf{A}_{m}, m \in L, m \geq l$.

Proof. By definition 14, for any $k \leq l$ and any $\mathbf{Q} \in \mathbf{A}_{k}$, there is a $b$ such that $b(\mathbf{Q}) \in \mathbf{A}_{l}$ and $b(\mathbf{Q}) \subseteq \mathbf{Q}$. Thus, if $\left\langle\mathfrak{T}_{n}, \mathbf{E}_{n}\right\rangle$ is epistemically empirically adequate for $\mathbf{A}_{l}$, there is some $n \in N$ such that for every $\mathbf{Q} \in \mathbf{A}_{k}$, there are a $\mathfrak{A} \in b(\mathbf{Q}) \subseteq \mathbf{Q}$ and an $\mathfrak{E} \in \mathbf{E}_{n}$ with $\mathfrak{E} \cong \mathfrak{A}$. The proof of the claim's second conjunct is similar. 
Claim 6. $\left\langle\left\{\mathbf{A}_{l}\right\}_{l \in L}, C\right\rangle$ is a (restricted) bierarchy of approximate appearances if and only if the following holds: For any $l \leq m$ with $l, m \in L$, there is an injection (bijection) $b: \mathbf{A}_{l} \longrightarrow \mathbf{A}_{m}$ in $C$ such that for all $\mathbf{Q} \in \mathbf{A}_{l}$ with $\left\{R_{i}^{+}, R_{i}^{-}, F_{j}^{+\circ}, c_{k}^{+\circ}\right\}_{i \in I, j \in J, k \in K}$ and for $b(\mathbf{Q}) \in \mathbf{A}_{m}$ with $\left\{\tilde{R}_{i}^{+}, \tilde{R}_{i}^{-}, \tilde{F}_{j}^{+\circ}, \tilde{c}_{k}^{+\circ}\right\}_{i \in I, j \in J, k \in K}$ it holds that $R_{i}^{+} \subseteq \tilde{R}_{i}^{+}, R_{i}^{-} \subseteq \tilde{R}_{i}^{-}, \tilde{F}_{j}^{+\circ} \subseteq F_{j}^{+\circ}$, and $\tilde{c}_{j}^{+\circ} \subseteq c_{j}^{+\circ}$ for all $i \in I, j \in$ $J, k \in K$.

Proof. ' $\Rightarrow$ ': By definition 14, there is an injection (bijection) $b: \mathbf{A}_{l} \longrightarrow \mathbf{A}_{m}$ in $C$ such that for all $\mathbf{Q}, b(\mathbf{Q}) \subseteq \mathbf{Q}$. The claim follows from (14)-(17).

$$
\text { ' } \Leftarrow \text { ': Immediate. }
$$

Claim 7. $\left\langle\mathfrak{T}_{n}, \mathbf{E}_{n}\right\rangle$ is epistemically/approximately empirically adequate at all points of all restricted hierarchies of epistemic/approximate appearances with the initial sequence $\left\langle\mathbf{A}_{l}\right\rangle_{l \leq m}$ if and only if $\left\langle\mathfrak{T}_{n}, \mathbf{E}_{n}\right\rangle$ is empirically adequate for all appearances that are epistemically possible given $\mathbf{A}_{m}$.

Proof. ' $\Rightarrow$ ': Assume $\left\langle\mathfrak{T}_{n}, \mathbf{E}_{n}\right\rangle$ is restrictedly epistemically/approximately adequate at all points of all hierarchies of epistemic appearances with the initial sequence $\left\langle\mathbf{A}_{l}\right\rangle_{l \leq m}$. For all appearances $\mathbf{A}$ that are epistemically possible given $\mathbf{A}_{l}$, the sequence $\left\langle\mathbf{A}_{m}, \mathbf{A}^{\prime}\right\rangle_{l \leq m}$ with $\mathbf{A}^{\prime}=\{\{\mathfrak{A}\}: \mathfrak{A} \in \mathbf{A}\}$ as its last element is a hierarchy of epistemic/approximate appearances. Therefore $\left\langle\mathfrak{T}_{n}, \mathbf{E}_{n}\right\rangle$ is epistemically empirically adequate for $\mathbf{A}^{\prime}$, and thus, by claim $4,\left\langle\mathfrak{T}_{n}, \mathbf{E}_{n}\right\rangle$ is empirically adequate for $\mathbf{A}$.

' $\Leftarrow$ ': For any point $\mathbf{A}_{r}$ of any hierarchy with initial sequence $\left\langle\mathbf{A}_{l}\right\rangle_{l \leq m}$, there is a bijection $b: \mathbf{A}_{m} \longrightarrow \mathbf{A}_{r}$ in $C$ with $b(\mathbf{Q}) \subseteq \mathbf{Q}$. By assumption, there is therefore a function $e$ from $\mathbf{A}_{r}$ to $\bigcup \mathbf{A}_{r}$ with $e(\mathbf{Q}) \in \mathbf{Q} \subseteq b^{-1}(\mathbf{Q})$ such that $\left\langle\mathfrak{T}_{n}, \mathbf{E}_{n}\right\rangle$ is empirically adequate for $\left\{e(\mathbf{Q}): \mathbf{Q} \in \mathbf{A}_{r}\right\}$. Since $\left\{e(\mathbf{Q}): \mathbf{Q} \in \mathbf{A}_{r}\right\}$ is epistemically possible given $\mathbf{A}_{r}$, by claim $3,\left\langle\mathfrak{T}_{n}, \mathbf{E}_{n}\right\rangle$ is epistemically/approximately empirically adequate for $\mathbf{A}_{r}$.

Claim 8. $\left\langle\mathfrak{T}_{n}, \mathbf{E}_{n}\right\rangle$ is at least as (restrictedly) epistemically empirically adequate as $\left\langle\mathfrak{T}_{s}, \mathbf{E}_{s}\right\rangle$ for any (restricted) hierarchy of epistemic appearances if and only if $\left\langle\mathfrak{T}_{n}, \mathbf{E}_{n}\right\rangle$ is empirically adequate for all appearances $\mathbf{A}$ for which $\left\langle\mathfrak{T}_{s}, \mathbf{E}_{s}\right\rangle$ is empirically adequate.

Proof. ' $\Rightarrow$ ': Choose the trivial (restricted) hierarchy of epistemic appearances $\langle\mathrm{A}\rangle$ with $\mathbf{A}$ containing all appearances for which $\left\langle\mathfrak{T}_{s}, \mathbf{E}_{s}\right\rangle$ is empirically adequate. Then all these appearances are epistemically possible appearances given $\mathbf{A}$, and by claim 3, the claim follows.

' $\Leftarrow$ ': Immediate from the definitions and claim 3 .

Claim 9. $\left\langle\mathfrak{T}_{s}, \mathbf{E}_{s}\right\rangle$ is empirically adequate for all appearances $\mathbf{A}$ for which $\left\langle\mathfrak{T}_{n}, \mathbf{E}_{n}\right\rangle$ is empirically adequate if and only if for every $n \in N$, there is an $s \in S$ such that all empirical substructures of $\mathfrak{T}_{n}$ are isomorphic to empirical substructures of $\mathfrak{T}_{s}$. 
Proof. ' $\Rightarrow$ ': For each $\mathbf{E}_{n}$, choose $\mathbf{A}=\mathbf{E}_{n}$. Then $\left\langle\mathfrak{T}_{n}, \mathbf{E}_{n}\right\rangle$ is empirically adequate for $\mathbf{A}$, and thus $\left\langle\mathfrak{T}_{s}, \mathbf{E}_{s}\right\rangle$ is empirically adequate for $\mathbf{A}$. Therefore for every $\mathfrak{E} \in$ $\mathbf{E}_{n}=\mathbf{A}$ there is an $s \in S$ and an $\mathfrak{E}^{\prime} \in \mathbf{E}_{s}$ such that $\mathfrak{E} \cong \mathfrak{E}^{\prime}$.

' $\Leftarrow$ ': Assume that for some $\mathbf{A},\left\langle\mathfrak{T}_{s}, \mathbf{E}_{s}\right\rangle$ but not $\left\langle\mathfrak{T}_{n}, \mathbf{E}_{n}\right\rangle$ is empirically adequate. Then there is an $s \in S$ such that for all $\mathfrak{A} \in \mathbf{A}$, there is an $\mathfrak{E}^{\prime} \in \mathbf{E}_{s}$ with $\mathfrak{A} \cong \mathfrak{E}^{\prime}$. Since there is no such $n \in N$ and the isomorphism relation is transitive, there is no $n$ such that for all $\mathfrak{E}^{\prime} \in \mathrm{E}_{s}$, there is an $\mathfrak{E} \in \mathrm{E}_{n}$ with $\mathfrak{E}^{\prime} \cong \mathfrak{E}$.

\section{References}

Batterman, Robert W. 2002. The Devil in the Details. Asymptotic Reasoning in Explanation, Reduction, and Emergence. Oxford Studies in Philosophy of Science. Oxford and New York: Oxford University Press.

Bell, J. L., and A. B. Slomson. 1974. Models and Ultraproducts: An Introduction. $3^{\text {rd }}$. Amsterdam: North-Holland.

Bueno, Otávio. 1997. "Empirical Adequacy: A Partial Structures Approach." Studies in the History and Philosophy of Science 28 (4): 585-610. doi:10.1016/ S0039-3681(97) 00012-5.

Bueno, Otávio, Steven French, and James Ladyman. 2002. "On Representing the Relationship between the Mathematical and the Empirical." Philosophy of Science 69 (3): 497-518. doi:10.1086/342456.

Carnap, Rudolf. 1939. Foundations of Logic and Mathematics. I,3:139-213. Foundations of the Unity of Science. Toward an International Encyclopedia of Unified Science. References are to the two-volume edition. Chicago and London: University of Chicago Press.

Chang, Chen Chung, and H. Jerome Keisler. 1990. Model Theory. $3^{\text {rd }}$. Studies in Logic and the Foundations of Mathematics. $3^{\text {rd }}$ impression 1992. Amsterdam: North Holland.

Da Costa, Newton, and Steven French. 1990. "The Model-Theoretic Approach in the Philosophy of Science." Philosophy of Science 57:248-65.

Fine, Kit. 1975. "Vagueness, Truth and Logic." References are to the corrected reprint (Fine 1997), Synthese 30 (3-4): 265-300.

. 1997. "Vagueness, Truth and Logic." In Vagueness. A Reader, edited by Rosanna Keefe and Peter Smith, 119-150. Cambridge, MA, and London: The MIT Press.

Van Fraassen, Bas C. 1970. "On the Extension of Beth's Semantics of Physical Theories." Philosophy of Science 37 (3): 325-39. 
Van Fraassen, Bas C. 1980. The Scientific Image. The Clarendon Library of Logic and Philosophy. Oxford: Clarendon Press.

1989. Laws and Symmetry. The Clarendon Library of Logic and Philosophy. Oxford: Clarendon Press.

- 1991. Quantum Mechanics: An Empiricist View. Oxford: Clarendon Press.

- 2002. The Empirical Stance. The Terry Lectures. New Haven, CT: Yale University Press.

- 2008. Scientific Representation: Paradoxes of Perspective. Oxford: Clarendon Press.

French, Steven, and James Ladyman. 1999. "Reinflating the Semantic Approach." International Studies in the Philosophy of Science 13 (2): 103-121. doi:10.1080/ 02698599908573612.

Friedman, Michael. 1982. "Review." Review of (van Fraassen 1980), The Journal of Philosophy 79 (5): 274-283.

Hodges, Wilfrid. 1993. Model Theory. Encyclopedia of Mathematics and its Applications. Digitally printed in 2008. Cambridge: Cambridge University Press.

Lutz, Sebastian. 2012. "On a Straw Man in the Philosophy of Science: A Defense of the Received View." HOPOS: The Journal of the International Society for the History of Philosophy of Science 2 (1): 77-120. doi:10.1086/664460.

- 2013. "The Semantics of Scientific Theories." Forthcoming. http:// philsci-archive.pitt.edu/id/eprint/9630.

- 2014a. "Empirical Adequacy in the Received View." Forthcoming. Preprint: http : / / philsci - archive - pitt . edu / id / eprint / 9869, Philosophy of Science.

- 2014b. "What's Right With a Syntactic Approach to Theories and Models?” Forthcoming, Erkenntnis. doi:10.1007/s10670-013-9578-5.

Monton, Bradley, and Chad Mohler. 2008. "Constructive Empiricism.” In The Stanford Encyclopedia of Philosophy, Winter 2008, edited by Edward N. Zalta. Stanford: The Metaphysics Research Lab, Center for the Study of Language / Information, Stanford University.

Muller, F. A. 1997. "The Equivalence Myth of Quantum Mechanics-Part II." Studies in the History and Philosophy of Modern Physics 28 (2): 219-241. doi:10. 1016/S1355-2198(97)00001-4. 
Percival, Philip. 2007. "An Empiricist Critique of Constructive Empiricism: The Aim of Science." Chap. 6 in Images of Empiricism. Essays on Science and Stances, with a Reply from Bas C. van Fraassen, edited by Bradley Monton. Mind Association Occasional Series. Oxford: Oxford University Press.

Przełęcki, Marian. 1969. The Logic of Empirical Theories. Monographs in Modern Logic Series. London/New York: Routledge \& Kegan Paul/Humanities Press.

1974a. "A Set Theoretic Versus a Model Theoretic Approach to the Logical Structure of Physical Theories." Studia Logica 33 (1): 91-105.

1974b. "On Model Theoretic Approach to Empirical Interpretation of Scientific Theories." Synthese 26:401-406.

1975. "Review of Ryszard Wójcicki: Metodologia formalna nank empirycznych. Podstawowe pojęcia $i$ zagadnienia (Formal Methodology of Empirical Sciences. Basic Concepts and Problems), Ossolineum, Wrocław, 1974." Studia Logica 34 (3): 275-284.

1976. "Fuzziness as Multiplicity." Erkenntnis 10:371-380.

Suárez, Mauricio. 2005. "The Semantic View, Empirical Adequacy, and Application." Crítica, Revista Hispanoamericana de Filosofia 37 (109): 29-63.

Suppes, Patrick. 1962. "Models of Data." In Logic, Methodology, and Philosophy of Science: Proceedings of the 1960 International Congress, edited by Ernest Nagel, Patrick Suppes, and Alfred Tarski, 252-261. Stanford: Stanford University Press.

Turney, Peter. 1990. "Embeddability, Syntax, and Semantics in Accounts of Scientific Theories." Journal of Philosophical Logic 19:429-451.

Volpe, Giorgio. 1995. "A Semantic Approach to Comparative Verisimilitude." The British Journal for the Philosophy of Science 46 (4): 563-581. doi:10.1093/ bjps/46.4.563.

Worrall, John. 1984. “An Unreal Image.” Review of (van Fraassen 1980), The British Journal for the Philosophy of Science 35:65-80. 